\title{
Reduced Order Computational Methods for Electromagnetic Material Interrogation Using Pulsed Signals and Conductive Reflecting Interfaces
}

\author{
H.T. Banks and G.M. Kepler \\ Center For Research in Scientific Computation \\ North Carolina State University \\ Raleigh, NC 27695-8205
}

Send correspondence and reprint requests to:

H.T. Banks, Director

Center For Research in Scientific Computation

Box 8205 North Carolina State University

Raleigh, NC 27695-8205

Telephone, 919-515-3968; Fax, 919-515-1636; Email,

htbanks@eos.ncsu.edu

\begin{abstract}
We consider the interrogation by means of a pulsed planar electromagnetic wave of a dielectric slab with a supraconductive backing. Previous work using a weak formulation with finite elements (FE) demonstrated the ability to determine material parameters and the slab thickness in the inverse problem. In this work we report on results using Proper Orthogonal Decomposition (POD) to create a more efficient set of basis functions than the standard FE basis functions. We first demonstrate the ability of the reduced basis POD formulation to capture the electromagnetic behavior in the case of the forward problem. We then apply the POD formulation to the inverse scattering problem with unknown parameters and show that the POD formulation provides a considerable reduction in computational time over standard FE methods with comparable ability to recover the unknown parameter values.
\end{abstract}




\section{INTRODUCTION}

Determination of material properties by means of a non-invasive probes such as low energy electromagnetic pulses is desirable in a broad range of applications in biology [1] and industry. Previous work has shown how metallic or other reflective backings could be exploited to obtain information about material properties and geometry [10]. In that work, a finite element (FE) based variational formulation was employed that incorporated Maxwell's full equations, the antenna source current, and constitutive polarization models (Debye or Lorentz). Application of the FE variational formulation to a 1D pulsed input scattering problem demonstrated the ability to capture the electromagnetic behavior in the forward problem. Using windowed microwave pulses in a $\mathrm{FE}$ variational formulation, the authors of [10] were able to effectively utilize the two reflected signals (from the air-dielectric interface and from the dielectric-backing interface) to estimate material dielectric properties and material thickness in the inverse problem.

Solution of the inverse problem to identify material properties can be time consuming, however, since it requires repeated calls to the forward finite element simulation, whose solution time grows as the square of the number of nodes. In an effort to reduce computational time we have implemented a reduced order formulation of the variational approach of Banks, et al.[10]. In place of the finite element basis functions our variational formulation uses basis elements obtained through application of the method of proper orthogonal decomposition (POD) to standard finite element computational results.

Our efforts reported on here were motivated by the very successful use of POD-based reduced order methods in other electromagnetic interrogation problems. In $[9,11]$ the authors considered eddy current based methods for interrogaton of dielectric materials for anomalies (flaws, damages, etc.). Through both computational and experimental validation efforts, they demonstrated the enormous potential for computational savings in this class of problems. These efforts, however, employed eddy currents originating from smooth (uniform) AC signals in conductive sheets. This permitted the reduction of the associated time domain Maxwell's equation system to a phasor form involving harmonic systems without transients. A question of great interest is whether the reduced order ideas of $[9,11]$ can be successfully employed in electromagnetic interrogation problems where the interrrogating signal is a microwave pulse and the resulting dielectric interface reflections/transmissions involve transients that must be accurately computed in order to perform the material identification characterization desired. In this paper we present the first evidence that this question can be answered in the affirmative.

Proper orthogonal decomposition, also known as principal component analysis [21] and Karhunen-Loève expansion [22, 26], is a well known method for feature extraction in statistical and pattern recognition fields [18]. The POD method has also been applied in a wide variety of other fields such as materials processing [12, 23, 24, 29, 35], characterization of human faces [33], and turbulent coherent flows ([4, 14, $15,16,19,27,34]$ - see also the surveys $[13,28])$.

The POD method linearly transforms a multivariate data set into an optimal set of uncorrelated variables (POD modes). The original multivariate data can be written as linear combinations of the POD modes. In many cases the POD modes more efficiently describe the variability of the original data and some dimensional reduction is possible by retaining only the most important modes.

Recent use of POD for reduction of order in distributed parameter systems includes as noted above applications to parameter estimation or inverse problems $[9,11]$ as well as applications to both open loop and feedback control design $[2,3,7,8,12,23,24,25,29,30,35]$. Computational evidence from a number of fluid and electromagnetic applications $[12,9,11,24,23]$ indicates that the important features 
of these systems are essentially of low finite dimension and amenable to approximation by a finite span of appropriately chosen basis elements.

We apply our POD reduced order model to a $1 \mathrm{D}$ scattering problem in which a windowed microwave pulse is incident on a dielectric slab with a supraconductive backing. We present results for the forward problem which show that the reduced order model is capable of capturing the electromagnetic behavior using significantly fewer modes than the FEM. We then apply the reduced order model to the inverse problem and show that the reduced order model is capable of accurately estimating the parameters with a significant reduction in computational time over the standard FEM. We believe that this work demonstrates the feasibility of utilizing the POD reduced order variational method for parameter estimation in transient electromagnetic systems when computational time is an important consideration.

\section{Problem Formulation and Approximations}

The physical problem. Consider the problem of interrogating an infinite (in the $x$ and $y$ directions) slab of homogeneous material by a windowed microwave pulse (Fig. 1). The interrogating signal is chosen to be a polarized planar electromagnetic wave normally incident on an infinite slab of material contained in the interval $\left[z_{1}, z_{2}\right]$ with faces parallel to the $x y$ plane. The electric field is polarized with oscillations in the $x z$ plane only. The slab will be denoted by $\Omega$ and the region exterior to the slab by $\Omega_{0}$.

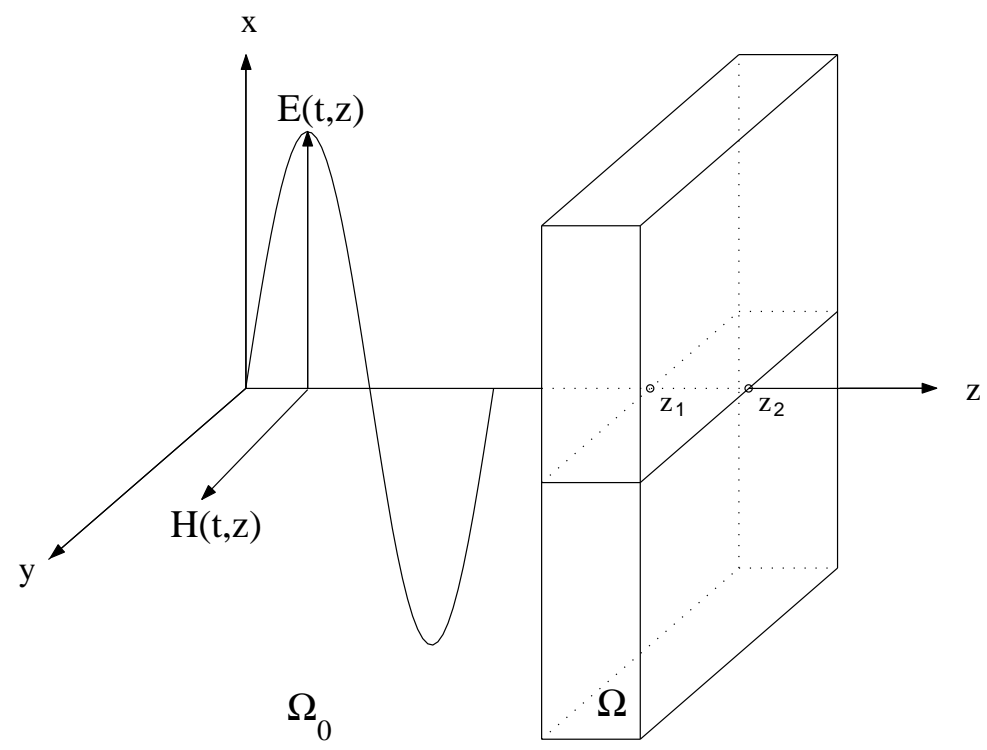

Figure 1: Geometry of the 1D scattering problem.

The electric and magnetic fields in $\Omega$ and $\Omega_{0}$ are governed by the macroscopic Maxwell's equations (see, 
for example, [20])

$$
\begin{aligned}
\nabla \times \vec{E} & =-\frac{\partial \vec{B}}{\partial t}, \\
\nabla \times \vec{H} & =\frac{\partial \vec{D}}{\partial t}+\vec{J} \\
\nabla \cdot \vec{D} & =\rho, \\
\nabla \cdot \vec{B} & =0 .
\end{aligned}
$$

The vector-valued functions $\vec{E}$ and $\vec{H}$ represent the strengths of the electric and magnetic fields, respectively, while $\vec{D}$ and $\vec{B}$ are the electric and magnetic fluxes, respectively. The conduction and source current densities are represented by $\vec{J}_{c}$ and $\vec{J}_{s}$, respectively. The electric and magnetic polarizabilities are represented by $\vec{P}$ and $\vec{M}$, respectively. The electric and magnetic susceptibilities are represented by $\epsilon_{0}$ and $\mu_{0}$, respectively and the density of free electric charge is represented by $\rho$.

The domain $\Omega_{0}$ is treated as empty space. As such, $\vec{M}=0, \vec{P}=0$, and $\vec{J}_{c}=0$. In addition, it is assumed that all parameters necessary for the determination of the fields are known in this region. The source current $\vec{J}_{s}$ is generated by an infinite antenna along the $x$ axis and hence will only be nonzero at these points in $\Omega_{0}$; its time-varying component will generate the interrogating electromagnetic waves.

For the purposes of this study, we will make certain assumptions about the material properties of the domain $\Omega$, neglecting magnetic effects and assuming that Ohm's law governs the electrical conductivity. Thus we have

$$
\left.\begin{array}{rl}
\vec{M}(\vec{x}) & =0 \\
\overrightarrow{J_{c}}(\vec{x}) & =\sigma \vec{E}(\vec{x})
\end{array}\right\} \quad \vec{x} \in \Omega
$$

Polarization model. For simplicity, we treat the instantaneous polarization to be proportional to the electric field so that $\vec{P}_{i n}=\epsilon_{0} \chi \vec{E}$, where $\chi$ is a dielectric constant. The electric flux density in (2.1) then can be written as

$$
\begin{aligned}
\vec{D} & =\epsilon_{0} \vec{E}+\epsilon_{0} \chi \vec{E}+\vec{P} \\
& =\epsilon_{0}(1+\chi) \vec{E}+\vec{P} \\
& =\epsilon_{0} \epsilon_{r} \vec{E}+\vec{P},
\end{aligned}
$$

where $\epsilon_{r}=1+\chi \geq 1$ is the relative permeability. The parameter $\epsilon_{r}$ is a spatially-dependent parameter that allows for instantaneous effects on the the displacement in $\Omega$ due to the electric field originating in $\Omega_{0}$. The remainder of the macroscopic electric polarizability of the medium $\Omega$ is denoted by $\vec{P}$ and is represented as an integral representation dependent upon a dielectric response function (DRF) and the past history of the electric field (for a discussion of this model and its relationship to other models in the literature, see [10], pg. 10)

$$
\vec{P}(t, \vec{x})=\int_{0}^{t} g(t-s, \vec{x}) \vec{E}(s, \vec{x}) d s
$$

where it is assumed that $\vec{P}(0, \vec{x})=0$.

In particular, we choose the Debye model $[10,17]$ for orientational or dipolar polarization in $\Omega$ described by

$$
\begin{aligned}
\tau \dot{\vec{P}}+\vec{P} & =\epsilon_{0}\left(\epsilon_{s}-\epsilon_{\infty}\right) \vec{E} \\
\vec{D} & =\epsilon_{\infty} \epsilon_{0} \vec{E}+\vec{P}
\end{aligned}
$$

where $\epsilon_{s}$ is the static relative permeability. The Debye model corresponds to $g(t)=e^{-t / \tau} \epsilon_{0}\left(\epsilon_{s}-\epsilon_{\infty}\right) / \tau$ in (2.4) and models the behavior of materials whose molecules possess permanent dipole moments. 
The magnitude of the polarization $\vec{P}$ represents the degree of alignment of the individual moments. Comparing (2.5) and (2.3), one finds that

$$
\epsilon_{r}=\left\{\begin{array}{cc}
\epsilon_{\infty} & \text { in } \Omega \\
1 & \text { in } \Omega_{0}
\end{array}\right.
$$

for the Debye polarization model.

Mathematical formulation. Using (2.1) and the condition of a plane-polarized interrogating input signal, one can argue (see [10]) that $\vec{E}(t, \vec{x})=\hat{i} E(t, z)$ and $\vec{H}(t, \vec{x})=\hat{j} H(t, z)$ for scalars $E$ and $H$ in $\Omega_{0}$. As a result, the polarizabilty $\vec{P}$ and flux density $\vec{D}$ are confined to the $x z$ plane (with nonzero components only in the $x$ direction) and can be denoted by scalar values $P$ and $D$. Since the material $\Omega$ is homogeneous in the $x y$ direction, the fields can be represented by scalar values $E, H, P$, and $D$ in $\Omega$ as well. Recalling that $\vec{J}_{s}$ also lies in the $x$ direction we find that Maxwell's equations then reduce to

$$
\begin{aligned}
\frac{\partial E}{\partial z} & =-\mu_{0} \frac{\partial H}{\partial t} \\
-\frac{\partial H}{\partial z} & =\frac{\partial D}{\partial t}+\sigma E+J_{s} .
\end{aligned}
$$

By taking appropriate derivatives, we can combine the equations (2.7) to give

$$
\mu_{0} \epsilon \ddot{E}+\mu_{0} \ddot{P}+\mu_{0} \sigma \dot{E}-E^{\prime \prime}=-\mu_{0} \dot{J}_{s},
$$

where (2.3) was used to eliminate $D, \epsilon=\epsilon_{0}\left(1+\left(\epsilon_{r}-1\right) I_{\Omega}\right), I_{S}$ denotes an indicator function for set $S$ (i.e., $I_{S}=1$ in $S$ and 0 otherwise), ${ }^{\prime}=\frac{\partial}{\partial z}$, and ${ }^{\circ}=\frac{\partial}{\partial t}$.

Boundary conditions. An absorbing boundary condition is placed at $z=0$ to prevent the reflection (back into the region of interest) of waves so that

$$
\left[\frac{1}{c} \frac{\partial E}{\partial t}-\frac{\partial E}{\partial z}\right]_{z=0}=0
$$

where $c^{2}=1 / \epsilon_{0} \mu_{0}$. The boundary conditions at the slab-supraconductive backing boundary are $\vec{B} \cdot \hat{n}=0$, which is automatically satisfied, since $\vec{B}=\hat{j} B$, and the condition $\vec{E} \times \hat{n}=0$, which gives $\left|E_{y} \hat{i}-E_{x} \hat{j}\right|_{\Gamma}=0$, so that $E_{x}=E_{y}=0$ on the boundary. This is equivalent to $E(t, 1)=0$ in our system. In addition, the source current $\vec{J}_{s}$ is specified at $z=0$ (as we have noted along the $\mathrm{x}$-axis).

Method of mappings. While the value of $z_{1}$ is known, the value of $z_{2}$ is generally unknown. We use a piece-wise linear mapping that leaves the interval $\left(0, z_{1}\right)$ invariant and maps $\left(z_{1}, z_{2}\right)$ to $\left(z_{1}, 1\right)$. The effect of this mapping $[5,6,10,31]$ is to transform the original unknown geometry $\Omega_{0} \bigcup \Omega$ to one with a known geometry $\tilde{\Omega}=[0,1]$. A new coordinate variable in $\tilde{\Omega}=[0,1]$ can be defined as

$$
\tilde{z}=f(z)=\left\{\begin{array}{cl}
z, & 0<z<z_{1}, \\
z_{1}+\left(z-z_{1}\right) \frac{1-z_{1}}{z_{2}-z_{1}}, & z_{1} \leq z \leq z_{2} .
\end{array}\right.
$$

This can also be written as

$$
f(z)=z+(\zeta-1)\left(z-z_{1}\right) I_{\left[z_{1}, z_{2}\right]}(z)
$$

where $\zeta=\frac{1-z_{1}}{z_{2}-z_{1}}$ and $f^{\prime}(z)=1+(\zeta-1) I_{\Omega}(z)$. The chain rule is used to convert spatial derivatives to derivatives in terms of the new variable $\tilde{z}$. That is,

$$
\frac{\partial}{\partial z}=\frac{\partial \tilde{z}}{\partial z} \frac{\partial}{\partial \tilde{z}}=f^{\prime}(z) \frac{\partial}{\partial \tilde{z}} .
$$


Using $d \tilde{z}=f^{\prime}(z) d z$, one can write the inner products of the weak form as

$$
\begin{aligned}
\langle\phi, \psi\rangle & =\int_{0}^{z_{2}} \phi(z) \psi(z) d z \\
& =\int_{0}^{1} \frac{\tilde{\phi}(\tilde{z}) \tilde{\psi}(\tilde{z})}{1+(\zeta-1) I_{\Omega}} d \tilde{z} \\
\left\langle\phi^{\prime}, \psi^{\prime}\right\rangle & =\int_{0}^{z_{2}} \phi^{\prime}(z) \psi^{\prime}(z) d z \\
& =\int_{0}^{1}\left(1+(\zeta-1) I_{\Omega}\right) \tilde{\phi}^{\prime}(\tilde{z}) \tilde{\psi}^{\prime}(\tilde{z}) d \tilde{z}
\end{aligned}
$$

Forward or simulation problem. Consider the case of a Debye medium $\Omega$ with a supraconductive backing and $\epsilon_{r}(z)=\epsilon_{\infty}$ defining the instantaneous polarization in $\Omega$. We first express (2.8) in an alternate form by integrating it against a "test" function $\phi$, obtaining

$$
\begin{aligned}
\left\langle\mu_{0} \epsilon \ddot{E}, \phi\right\rangle & +\left\langle\mu_{0} \sigma \dot{E}, \phi\right\rangle+\left\langle\mu_{0} \ddot{P}, \phi\right\rangle \\
& -\left\langle E^{\prime \prime}, \phi\right\rangle=-\left\langle\mu_{0} \dot{J}_{s}(t, \cdot), \phi\right\rangle
\end{aligned}
$$

where the polarization $P$ is of the form (2.4) and the mapping to $\tilde{\Omega}=[0,1]$ has already been carried out. Integration by parts of (2.15) gives the weak form

$$
\begin{aligned}
\left\langle\mu_{0} \epsilon \ddot{E}, \phi\right\rangle & +\left\langle\mu_{0} \sigma \dot{E}, \phi\right\rangle+\left\langle\mu_{0} \ddot{P}, \phi\right\rangle \\
& +\left\langle E^{\prime}, \phi^{\prime}\right\rangle+\frac{1}{c} \dot{E}(t, 0) \phi(0)=-\left\langle\mu_{0} \dot{J}_{s}(t, \cdot), \phi\right\rangle,
\end{aligned}
$$

where the term $\frac{1}{c} \dot{E}(t, 0) \phi(0)$ is part of the weak form resulting from the absorbing boundary condition (2.9).

For computational purposes the time variable is scaled by a factor of $c=1 / \sqrt{\epsilon_{0} \mu_{0}}(\tilde{t}=c t)$ and the polarization $P$ by a factor of $1 / \epsilon_{0}\left(\tilde{P}=P / \epsilon_{0}\right)$. We assume that the electric permittivity and magnetic permeability of the medium $\Omega$ are constant. The scaled equation becomes

$$
\begin{aligned}
\left\langle\tilde{\epsilon}_{r} \ddot{E}, \phi\right\rangle & +\eta_{0}\langle\sigma \dot{E}, \phi\rangle+\langle\ddot{P}, \phi\rangle \\
& +\left\langle E^{\prime}, \phi^{\prime}\right\rangle+\dot{E}(t, 0) \phi(0)=-\eta_{0}\left\langle\dot{J}_{s}(t, \cdot), \phi\right\rangle,
\end{aligned}
$$

where $\tilde{\epsilon}_{r}(z)=1+I_{\Omega}(z)\left(\epsilon_{\infty}-1\right)$ is the relative electric permittivity so that $\epsilon=\tilde{\epsilon}_{r} \epsilon_{0}$ and the impedance of free space is defined $\eta_{0}=\sqrt{\mu_{0} / \epsilon_{0}} \approx 376.73 \mathrm{Ohms}$. The inner products $\langle\cdot, \cdot\rangle$ are the weighted inner products of (2.13).

FEM discretization. The interval $[0,1]$ is uniformly divided at the points $\tilde{z}_{j}^{N}=i h$, where $h=1 / N$ and $i=0, \ldots, N$. The electric and polarization fields $E$ and $P$ are discretized with $N$ piece-wise linear spline functions $\phi_{i}^{N}(\tilde{z})$ such that $\phi_{i}^{N}\left(\tilde{z}_{j}^{N}\right)=\delta_{i j}$ for $i, j=0, \ldots, N$. The essential boundary condition $\phi_{i}^{N}(1)=0$ is satisfied by omitting $\phi_{N}^{N}$ in the finite dimensional approximating subspace 
$V^{N}=\operatorname{span}\left\{\phi_{0}^{N}, \phi_{1}^{N}, \ldots, \phi_{N-1}^{N}\right\}$. The fields are then approximated by

$$
\begin{aligned}
& E(t, z) \approx E^{N}(t, z)=\sum_{i=0}^{N-1} e_{i}^{N}(t) \phi_{i}^{N}(z), \\
& P(t, z) \approx P^{N}(t, z)=\sum_{i=0}^{N-1} p_{i}^{N}(t) \phi_{i}^{N}(z),
\end{aligned}
$$

In order to avoid computational difficulties, we make one further requirement that the material boundaries of the slab $\left[z_{1}, 1\right]$ coincide with grid points. The grid point coinciding with the material boundary $z_{1}$ will be denoted by $j=L$. By design, the right edge of the material has been mapped to $z=1$, which corresponds to the grid point $j=N$.

Substitution of the approximations (2.18) into (2.17) yields

$$
\begin{aligned}
\sum_{i=0}^{N-1} \ddot{e}_{i}^{N}(t)\left\langle\tilde{\epsilon}_{r} \phi_{i}^{N}, \phi\right\rangle & +\sum_{i=0}^{N-1} \eta_{0} \dot{e}_{i}^{N}(t)\left\langle\sigma \phi_{i}^{N}, \phi\right\rangle+\sum_{i=0}^{N-1} \ddot{p}_{i}^{N}(t)\left\langle\phi_{i}^{N}, \phi\right\rangle \\
& +\sum_{i=0}^{N-1} e_{i}^{N}(t)\left\langle\phi_{i}^{N \prime}, \phi^{\prime}\right\rangle+\sum_{i=0}^{N-1} \dot{e}_{i}^{N}(t) \phi_{i}^{N}(0) \phi(0)=-\eta_{0}\left\langle\dot{J}_{s}(t, \cdot), \phi\right\rangle
\end{aligned}
$$

which can be written as

$$
\left(M^{N}+M_{\Omega}^{N}\left(\epsilon_{\infty}-1\right)\right) \ddot{e}^{N}+\left(\eta_{0} \sigma M_{\Omega}^{N}+B^{N}\right) \dot{e}^{N}+M_{\Omega}^{N} \ddot{p}^{N}+K^{N} e^{N}=\eta_{0} \mathcal{J}^{N}
$$

for $e=\left(e_{0}^{N}, e_{2}^{N}, \ldots, e_{N-1}^{N}\right)$ and $p=\left(p_{0}^{N}, p_{2}^{N}, \ldots, p_{N-1}^{N}\right)$. The elements for the $N \times N$ matrices are given by

$$
\begin{aligned}
M_{\Omega i j}^{N} & =\left\langle I_{\Omega} \phi_{i}, \phi_{j}\right\rangle=\int_{0}^{1} \frac{I_{\Omega} \tilde{\phi}_{i} \tilde{\phi}_{j}}{1+(\zeta-1) I_{\Omega}} d \tilde{z} \\
M_{i j}^{N} & =\left\langle\phi_{i}, \phi_{j}\right\rangle=\int_{0}^{1} \frac{\tilde{\phi}_{i} \tilde{\phi}_{j}}{1+(\zeta-1) I_{\Omega}} d \tilde{z} \\
B_{i j}^{N} & =\phi_{i}(0) \phi_{j}(0) \\
K_{i j}^{N} & =\left\langle\phi_{i}^{\prime}, \phi_{j}^{\prime}\right\rangle=\int_{0}^{1}\left(1+(\zeta-1) I_{\Omega}\right) \tilde{\phi}_{i}^{\prime} \tilde{\phi}_{j}^{\prime} d \tilde{z}
\end{aligned}
$$

while the $N \times 1$ finite element vector $\mathcal{J}^{N}$ is given by

$$
\mathcal{J}_{i}^{N}=-\left\langle\dot{J}_{s}(t, \cdot), \phi_{i}\right\rangle=-\int_{0}^{1} \frac{\dot{J}_{s} \tilde{\phi}_{i}}{1+(\zeta-1) I_{\Omega}} d \tilde{z}
$$

Note that the integrals are in terms of the scaled variables of (2.10).

We will use the Debye polarization model (2.5) to provide the constitutive law relating the polarization $P$ to the electric field $E$. Applying the same time scaling as above $\left(\tilde{P}=P / \epsilon_{0}, \tilde{t}=c t\right)$ we obtain

$$
\dot{P}+\lambda P=\epsilon_{d} \lambda E \text { in } \Omega,
$$

where $\epsilon_{d}=\epsilon_{s}-\epsilon_{\infty}$ and $\lambda=1 / c \tau$. To generalize the above equation to the entire domain we can multiply the equation by the indicator function $I_{\Omega}(z)$. Then, applying a Galerkin approximation, we obtain

$$
M_{\Omega}^{N} \dot{p}^{N}+M_{\Omega}^{N} \lambda p^{N}-M_{\Omega}^{N} \lambda \epsilon_{d} e^{N}=0
$$


The combined system of equations is

$$
\begin{aligned}
\left(M^{N}+M_{\Omega}^{N}\left(\epsilon_{\infty}-1\right)\right) \ddot{e}^{N}+M_{\Omega}^{N} \ddot{p}^{N}+\left(\eta_{0} \sigma M_{\Omega}^{N}+B\right) \dot{e}^{N}+K^{N} e^{N} & =\eta_{0} \mathcal{J}^{N} \\
\dot{p}^{N}+\lambda p^{N}-\lambda \epsilon_{d} e^{N} & =0 .
\end{aligned}
$$

Substituting the second equation and its derivative into the first, one finds

$$
\begin{aligned}
\left(M^{N}+M_{\Omega}^{N}\left(\epsilon_{\infty}-1\right)\right) \ddot{e}^{N} & +\left(\lambda \epsilon_{d} M_{\Omega}^{N}+\eta_{0} \sigma M_{\Omega}^{N}+B\right) \dot{e}^{N} \\
+ & \left(-\lambda^{2} \epsilon_{d} M_{\Omega}^{N}+K^{N}\right) e^{N}+\lambda^{2} M_{\Omega} p^{N}=\eta_{0} \mathcal{J}^{N} \\
& \dot{p}^{N}+\lambda p^{N}-\lambda \epsilon_{d} e^{N}=0
\end{aligned}
$$

This can be written as a first-order system with the composite variable $x=\left(e^{N}, p^{N}, \dot{e}^{N}\right)$ as

$$
\bar{M}^{3 N} \dot{x}+\bar{K}^{3 N} x=F^{3 N}
$$

or

$$
\left[\begin{array}{lll}
I & & \\
& I & \\
& & M_{1}^{N}
\end{array}\right]\left[\begin{array}{c}
e^{N} \\
p^{N} \\
\dot{e}^{N}
\end{array}\right]+\left[\begin{array}{ccc}
0^{N} & 0^{N} & -I^{N} \\
-\lambda \epsilon_{d} I_{L R}^{N} & \lambda I_{L R}^{N} & 0^{N} \\
M_{2}^{N} & M_{3}^{N} & M_{4}^{N}
\end{array}\right]\left[\begin{array}{c}
e^{N} \\
p^{N} \\
\dot{e}^{N}
\end{array}\right]=\left[\begin{array}{c}
0^{N} \\
0^{N} \\
\eta_{0} \mathcal{J}^{N}
\end{array}\right]
$$

where

$$
\begin{aligned}
& M_{1}^{N}=M^{N}+\left(\epsilon_{\infty}-1\right) M_{\Omega}^{N}, \\
& M_{2}^{N}=-\lambda^{2} \epsilon_{d} M_{\Omega}^{N}+K^{N}, \\
& M_{3}^{N}=\lambda^{2} M_{\Omega}^{N}, \\
& M_{4}^{N}=M_{\Omega}^{N}\left(\lambda \epsilon_{d}+\eta_{0} \sigma\right)+B^{N},
\end{aligned}
$$

and $I_{L R}$ is the $N \times N$ identity matrix where the ones have been replaced with zeros in rows 1 through $L-1$.

The form of the source current is chosen to be

$$
J_{s}(t, z)=\delta(z) g_{s}(t) I_{\left(0, t_{f}\right)}(t)=-\delta(z) \sin (\omega t) I_{\left(0, t_{f}\right)}(t),
$$

where $\omega$ is a specified angular frequency of the input signal (and the carrier frequency of the resulting planar wave) and $\delta(z)$ is the Dirac distribution with infinite mass at $z=0$. The signal is truncated at finite time $t_{f}$ coinciding with $g_{s}(t)=0$ by the indicator function $I_{\left(0, t_{f}\right)}(t)$ to avoid complications arising from discontinuities in the input signal. As a result, $\omega t_{f}=n \pi$ for some positive even integer $n$. The duration of the pulse must be sufficiently short to distinguish reflections from the front and back of the medium, thus requiring that $t_{f} c<2\left(z_{2}-z_{1}\right)$. The current matrix then becomes

$$
\mathcal{J}^{N}=\left[\begin{array}{c}
\omega \cos (\omega t) I_{\left(0, t_{f}\right)}(t) \\
0^{N-1}
\end{array}\right]
$$

Solution method. We use the standard Crank-Nicholson scheme to find an approximate solution for the differential equation system (2.27). Rewriting (2.27) as

$$
\dot{x}=f(t, x)=\left(\bar{M}^{3 N}\right)^{-1}\left(F^{3 N}-\bar{K}^{3 N} x\right)
$$

and choosing a step size $k$, we make the iterative approximation

$$
x_{n+1}=x_{n}+k f_{n+\theta}
$$


where $x_{n} \approx x\left(t_{n}\right)=x(n k), f_{n+\theta}=(1-\theta) f\left(t_{n}, x_{n}\right)+\theta f\left(t_{n+1}, x_{n+1}\right)$, and for the Crank-Nicholson scheme $\theta=1 / 2$. This system can be solved directly for $x_{n+1}$

$$
x_{n+1}=x_{n}+k y_{n},
$$

where

$$
\left(\bar{M}^{3 N}+k \theta \bar{K}^{3 N}\right) y_{n}=F_{n+\theta}^{3 N}-\bar{K}^{3 N} x_{n},
$$

$F_{n+\theta}^{3 N}=(1-\theta) F_{n}^{3 N}+\theta F_{n+1}^{3 N}$, and $x_{0}=0$. Equation (2.35) can be solved by means of block-Gaussian elimination to a block upper-triangular system, LU factorization, and back-substitution.

Construction of POD modes. Simulation using the finite-element system (2.27) above provides a multivariate data set consisting of $2 K$ vectors

$$
X=\left\{E_{1}^{N} \ldots E_{K}^{N} \ldots P_{1}^{N} \ldots P_{K}^{N}\right\}
$$

representing the $N$ nodal values of $E(t)$ and $P(t)$ at $K$ time points during the simulation. This original data set $X$ is transformed to a new set of uncorrelated variables (POD modes) by

$$
W=\left\{w_{1}^{N}, w_{2}^{N}, \ldots, w_{2 K}^{N}\right\}=X \Phi,
$$

where the columns of $\Phi=\left\{\phi_{1}^{2 K}, \phi_{2}^{2 K}, \ldots, \phi_{2 K}^{2 K}\right\}$ are the eigenvectors of the product matrix $\left(X^{\prime} X\right) \phi_{i}^{2 K}=$ $\lambda_{i} \phi_{i}^{2 K}$, ranked, in descending order, with respect to the associated eigenvalue and the prime superscript denotes the transpose of the matrix. The POD modes $W$ are orthogonal $w_{i}^{N} \cdot w_{j}^{N}=\lambda_{i} \delta_{i, j}$, and the transformation of variables preserves the data variability

$$
\sum_{k=1}^{2 K}\left(X^{\prime} X\right)_{k k}=\sum_{k=1}^{2 K}\left(W^{\prime} W\right)_{k k}=\sum_{k=1}^{2 K} \lambda_{k} .
$$

Expansion of the original data $X$ in terms of the most significant POD modes minimizes the mean square error of a reduced basis representation[18]

$$
\left(X^{R}\right)_{i j}=\sum_{k=1}^{R}(W)_{i k}(\Phi)_{k j}^{\prime},
$$

where $R<2 K$. The most significant POD modes are those corresponding to the largest eigenvalues, since the ratio of an eigenvalue to the summation of eigenvalues, $\lambda_{k} / \sum_{j=1}^{K} \lambda_{j}$, gives the percentage of the mean square error unaccounted for by eliminating the corresponding POD mode $w_{k}^{N}$ in the reduced basis representation [18]. The best stopping point in the expansion (2.39) depends, in general, upon the application and various algorithms have been proposed[21].

POD discretization. A second discretization formulation produces the reduced basis model. In this case, we first use the POD modes $\left\{w_{k}^{N}\right\}$ to obtain the POD elements

$$
\Psi_{k}(z)=\sum_{i=1}^{N}\left(w_{k}\right)_{i} \psi_{i}(z), \quad k=1,2, \ldots, 2 K
$$

where the functions $\psi_{i}(z)$ are the finite element linear interpolation functions. The electric and polarization fields are approximated as linear combinations of the POD basis elements corresponding to the 
most significant POD modes

$$
\begin{aligned}
& E(t, z) \approx E^{R}(t, z)=\sum_{i=1}^{R} e_{i}^{R}(t) \Psi_{i}^{R}(z) \\
& P(t, z) \approx P^{R}(t, z)=\sum_{i=1}^{R} p_{i}^{R}(t) \Psi_{i}^{R}(z)
\end{aligned}
$$

where, in this case, $R<N$. Application of this approximation in (2.17) (in this case we use POD test functions $\left.\phi_{i}=\Psi_{i} \quad i=1,2, \ldots, R\right)$ yields a Galerkin-POD system

$$
\begin{aligned}
\sum_{i=1}^{R} \ddot{e}_{i}^{R}(t)\left\langle\tilde{\epsilon}_{r} \Psi_{i}^{R}, \Psi^{R}\right\rangle & +\sum_{i=1}^{R} \eta_{0} \dot{e}_{i}^{R}(t)\left\langle\sigma \Psi_{i}^{R}, \Psi^{R}\right\rangle+\sum_{i=1}^{R} \ddot{p}_{i}^{R}(t)\left\langle\Psi_{i}^{R}, \Psi^{R}\right\rangle \\
& +\sum_{i=1}^{R} e_{i}^{R}(t)\left\langle\Psi_{i}^{R \prime}, \Psi^{R \prime}\right\rangle+\sum_{i=1}^{R} \dot{e}_{i}^{R}(t) \Psi_{i}^{R}(0) \Psi^{R}(0)=-\eta_{0}\left\langle\dot{J}_{s}(t, \cdot), \Psi^{R}\right\rangle
\end{aligned}
$$

which can be written as

$$
\left(M^{R}+M_{\Omega}^{R}\left(\epsilon_{\infty}-1\right)\right) \ddot{e}^{R}+\left(\eta_{0} \sigma M_{\Omega}^{R}+B^{R}\right) \dot{e}^{R}+M_{\Omega}^{R} \ddot{p}^{R}+K^{R} e^{R}=\eta_{0} \mathcal{J}^{R}
$$

for $e=\left(e_{1}^{R}, e_{2}^{R}, \ldots, e_{R}^{R}\right), p=\left(p_{1}^{R}, p_{2}^{R}, \ldots, p_{R}^{R}\right)$, and $\left.\dot{e}=\dot{e}_{1}^{R}, \dot{e}_{2}^{R}, \ldots, \dot{e}_{R}^{R}\right)$. The elements for the $R \times R$ matrices are given by

$$
\begin{aligned}
M_{\Omega i j}^{R} & =\left\langle I_{\Omega} \Psi_{i}^{R}, \Psi_{j}^{R}\right\rangle=\int_{0}^{1} \frac{I_{\Omega} \tilde{\Psi}_{i}^{R} \tilde{\Psi}_{j}^{R}}{1+(\zeta-1) I_{\Omega}} d \tilde{z} \\
M_{i j}^{R} & =\left\langle\Psi_{i}^{R}, \Psi_{j}^{R}\right\rangle=\int_{0}^{1} \frac{\tilde{\Psi}_{i}^{R} \tilde{\Psi}_{j}^{R}}{1+(\zeta-1) I_{\Omega}} d \tilde{z} \\
B_{i j}^{R} & =\Psi_{i}^{R}(0) \Psi_{j}^{R}(0) \\
K_{i j}^{R} & =\left\langle\Psi_{i}^{R \prime}, \Psi_{j}^{R \prime}\right\rangle=\int_{0}^{1}\left(1+(\zeta-1) I_{\Omega}\right) \tilde{\Psi}_{i}^{R \prime} \tilde{\Psi}_{j}^{R \prime} d \tilde{z}
\end{aligned}
$$

while the $R \times 1$ finite element vector is

$$
\mathcal{J}_{i}^{R}=-\left\langle\dot{J}_{s}(t, \cdot), \Psi_{i}^{R}\right\rangle=-\int_{0}^{1} \frac{\dot{J}_{s} \tilde{\Psi}_{i}^{R}}{1+(\zeta-1) I_{\Omega}} d \tilde{z}
$$

and the integrals are in terms of the scaled variables defined in (2.10). Choosing the source current as in $(2.30)$ we find

$$
\mathcal{J}_{i}^{R}=\omega \cos (\omega t) I_{\left(0, t_{f}\right)}(t) \Psi_{i}^{R}(0)
$$

Application of the POD approximations to the Debye constitutive law (2.5) relating the polarization $P$ to the electric field $E$ gives

$$
M_{\Omega}^{R} \dot{p}^{R}+M_{\Omega}^{R} \lambda p^{R}-M_{\Omega}^{R} \lambda \epsilon_{d} e^{R}=0 .
$$

Since the POD basis functions $\Psi_{R}$ are global spatially distributed functions, the matrix $M_{\Omega}^{R}$ is not singular, which differs from the finite element formulation.

The combined system of equations is (2.43) and (2.47) is

$$
\begin{aligned}
\left(M^{R}+M_{\Omega}^{R}\left(\epsilon_{\infty}-1\right)\right) \ddot{e}^{R}+M_{\Omega}^{R} \ddot{p}^{R}+\left(\eta_{0} \sigma M_{\Omega}^{R}+B^{R}\right) \dot{e}^{R}+K^{R} e^{R} & =\eta_{0} \mathcal{J}^{R} \\
\dot{p}^{R}+\lambda p^{R}-\lambda \epsilon_{d} e^{R} & =0 .
\end{aligned}
$$


Substituting the second equation and its derivative into the first, we obtain

$$
\begin{aligned}
\left(M^{R}+M_{\Omega}^{R}\left(\epsilon_{\infty}-1\right)\right) \ddot{e}^{R}+ & \left(\lambda \epsilon_{d} M_{\Omega}^{R}+\eta_{0} \sigma M_{\Omega}^{R}+B^{R}\right) \dot{e}^{R} \\
+ & \left(-\lambda^{2} \epsilon_{d} M_{\Omega}^{R}+K^{R}\right) e^{R}+\lambda^{2} M_{\Omega}^{R} p^{R}=\eta_{0} \mathcal{J}^{R} \\
& I \dot{p}^{R}+\lambda I p^{R}-\lambda \epsilon_{d} I e^{R}=0,
\end{aligned}
$$

where $I$ is the $R \times R$ identity. This can be written as a first-order system with the $3 R$ vector variable $x=\left(e^{R}, p^{R}, \dot{e}^{R}\right)$ as

$$
\begin{gathered}
\bar{M}^{3 R} \dot{x}+\bar{K}^{3 R} x=F^{3 R} \\
{\left[\begin{array}{lll}
I & & \\
& I & \\
& & M_{1}^{R}
\end{array}\right]\left[\begin{array}{c}
e^{R} \\
p^{R} \\
\dot{e}^{R}
\end{array}\right]+\left[\begin{array}{ccc}
0^{R} & 0^{R} & -I^{R} \\
-\lambda \epsilon_{d} I & \lambda I & 0^{R} \\
M_{2}^{R} & M_{3}^{R} & M_{4}^{R}
\end{array}\right]\left[\begin{array}{c}
e^{R} \\
p^{R} \\
\dot{e}^{R}
\end{array}\right]=\left[\begin{array}{c}
0^{R} \\
0^{R} \\
\eta_{0} \mathcal{J}^{R}
\end{array}\right],}
\end{gathered}
$$

where

$$
\begin{aligned}
& M_{1}^{R}=M^{R}+\left(\epsilon_{\infty}-1\right) M_{\Omega}^{R} \\
& M_{2}^{R}=-\lambda^{2} \epsilon_{d} M_{\Omega}^{R}+K^{R} \\
& M_{3}^{R}=\lambda^{2} M_{\Omega}^{R}, \\
& M_{4}^{R}=M_{\Omega}^{R}\left(\lambda \epsilon_{d}+\eta_{0} \sigma\right)+B^{R}
\end{aligned}
$$

The solution to the differential equation system (2.50) can be approximated using a Crank-Nicholson scheme in the same manner as described above for the FEM differential equation system (2.27).

Inverse problem. We formulate the problem described in detail in [10] of the interrogation of a Debye dielectric medium with a supraconductive backing by a plane-polarized windowed wave. In the inverse problem we attempt to determine parameter values of the dielectric material from experimental measurements at $z=0$ of the reflected electric field. This is accomplished by minimizing the $L^{2}$ difference between the experimental data and simulation results

$$
\min _{\vec{q} \in Q} J(\vec{q})=\sum_{i=1}^{S}\left|E\left(\bar{t}_{i}, 0 ; \vec{q}\right)-\hat{E}_{i}\right|^{2},
$$

where $S$ is the number of sample data points $\hat{E}_{i}$, at uniform time intervals $\bar{t}_{i}, Q$ is the set of admissable parameters, and $E\left(\bar{t}_{i}, 0 ; \vec{q}\right)$ are the electic field values arising from simulations with parameters $\vec{q}$. The vector $\vec{q}$ typically contains dielectric and/or conductivity parameters characterizing the medium and/or geometric properties (see [10]).

For our computational testing of the methods and algorithms, synthetic data $\hat{E}_{i}$ is produced by adding random noise to the results of FEM simulations with a known set of parameters, i.e.,

$$
\hat{E}_{i}=E_{i}\left(1+\nu \eta_{i}\right),
$$

where $E_{i}$ are the values sampled from the solution with known parameters, $\eta_{i}$ are independent normally distributed random variables with mean zero and variance one [10]. The amplitude of the noise is proportional to the signal level $E_{i}$ and the coefficient $\nu$. By choice of $\nu$, we can control the relative noise. For example, $10 \%$ relative noise is achieved (with probability 0.9545 ) by choice of $\nu=0.05$ since $\eta_{i}$ lies in $[-2 \sigma, 2 \sigma]=[-2,2]$ (with probability 0.9545$)$.

Minimization of $J(\vec{q})$ is performed using a Broyden-Fletcher-Goldfarg-Shanno (BFGS) variable metric algorithm $(d f p \min ()$, pg. $428[32])$. The gradient of $J(\vec{q})$ is obtained from a forward-difference approximation using an algorithm adapted from (fdjac(), pg. 388 [32]). The BFGS algorithm and related 
functions are modified for double-precision. In addition, constraints are added to reflect the physical limitation of parameters, ie., no negative values. The convergence requirement for zeroing the gradient is $1 \times 10^{-4}$ for all the minimization problems reported here.

The values $E\left(\bar{t}_{i}, 0 ; \vec{q}\right)$ appearing in (2.53) are obtained from simulations using either FE or POD basis elements. Simulation using FE basis vectors is straight-forward, but slow, since the basis vectors are independent of the parameter values. Minimization of $J(\vec{q})$ using simulations with POD basis elements is faster, but more complicated, since the POD basis elements are generated from snapshots obtained from FE simulations with specified parameter values. For the minimization problem a collection of snapshots from simulations covering a range of parameter values $(\vec{q}))$ are used to generate the POD basis elements.

$$
X_{n K}^{N}=\left\{X_{2 K}^{N}\left(\vec{q}_{1}\right) X_{2 K}^{N}\left(\vec{q}_{2}\right) \ldots X_{2 K}^{N}\left(\vec{q}_{n}\right)\right\}
$$

where each snapshot represent $N$ nodal values and there are $K$ snapshots each for the electric and polarization fields per simulation. In general, the simulation results change gradually with changes in parameter values and the POD modes are still able to efficiently represent the range of data. 


\section{COMPUTATIONAL RESULTS}

Forward problems. We simulated the interrogation of a dielectric slab with a forward face at $z_{1}=0.33$ and a supraconductive backing at $z_{2}=0.89$ by a plane-polarized windowed wave using the techniques outlined above. The mapped region $\tilde{\Omega}$ was discretized with $N=450$ and $L=150$. The dielectric parameter values were $\epsilon_{s}=35, \epsilon_{\infty}=5, \tau=1 \times 10^{-11} \mathrm{~s}, \sigma=1.0 \times 10^{-2} \mathrm{Ohm}^{-1}$. The signal parameters were chosen to be: $\phi=1.8 \times 10^{9} \mathrm{~s}^{-1}, \omega=2 \pi \times \phi \mathrm{rad} / \mathrm{sec}$, and $t_{f}=\frac{6}{\phi}=3.33 \times 10^{-9} \mathrm{~s}$. The differential system was integrated with a step size of $d t=1.0 \times 10^{-4} \mathrm{~ns}$ for a total of $t_{\text {final }}=10 \mathrm{~ns}$.

We experimented with different snapshot intervals for the creation of the POD modes. POD modes created using a sample rate, or snapshot interval, of 0.005 ns provided an efficient representation of the data. Figure 2 shows the percent variability captured for the field data $(E(t)$ and $P(t))$ as a function of the number of modes at the $0.005 \mathrm{~ns}$ sampling interval. Some representative values are $99.099 \%$, $99.962 \%$, and $99.999 \%$ for 30,50 , and 72 modes, respectively.

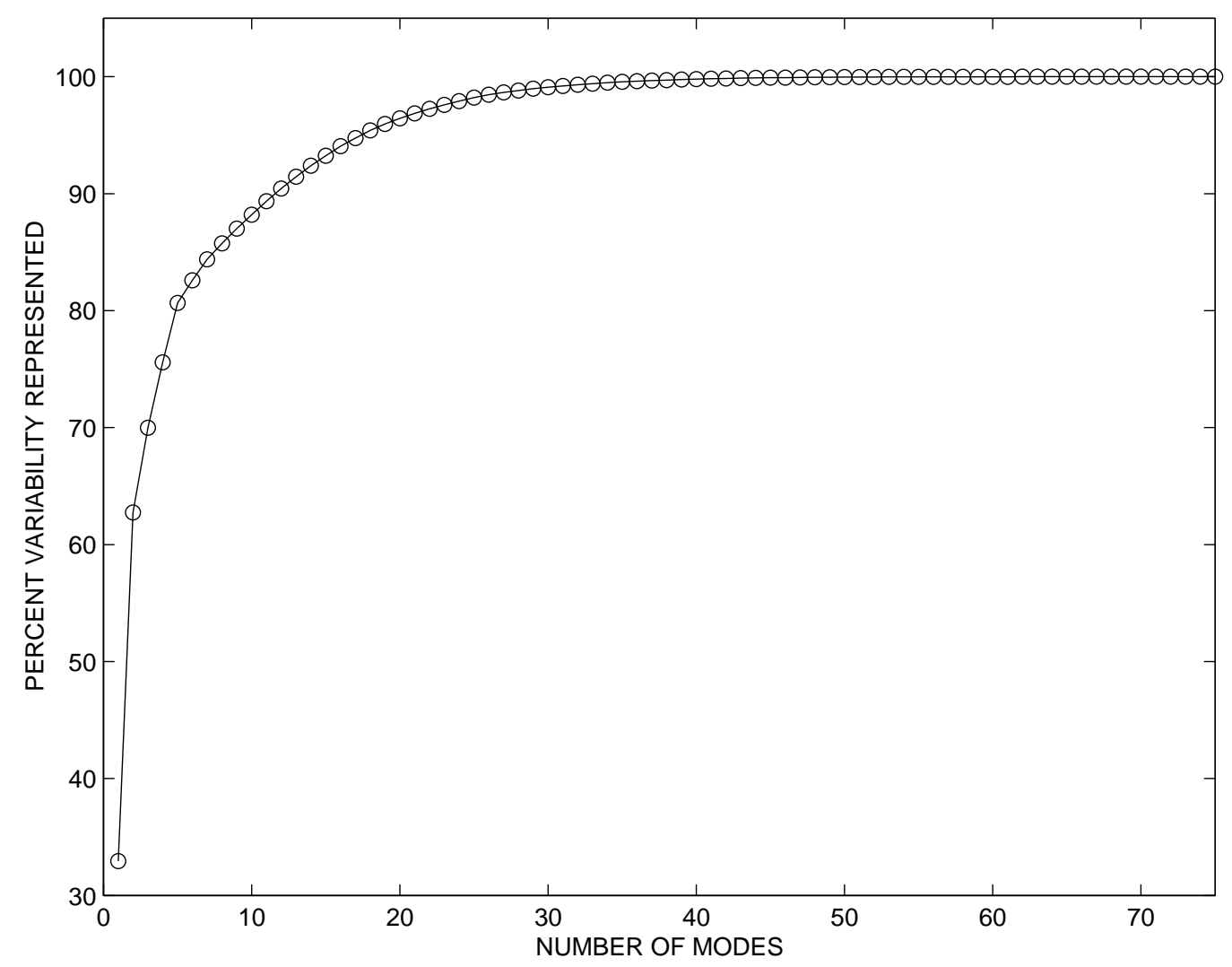

Figure 2: Percent of field $(E(t)$ and $P(t))$ variability represented as a function of the number of modes used. The sampling interval was $0.005 \mathrm{~ns}$, giving 2001 snapshots for each field. 
We illustrate the efficacy of the POD reduced order representation by simulating the forward problem using the first $72 \mathrm{POD}$ modes (of 4002 possible modes) generated from snapshots taken at the $0.005 \mathrm{~ns}$ sampling rate.

Figures 3-6 compare electric field results obtained using FEM ( $N=450$ and $\mathrm{L}=150)$ and POD $(\mathrm{R}=72)$ simulations. In Figure 3 the electric field is plotted as a function of the mapped distance $\mathrm{z}$ at $0.7 \mathrm{~ns}$. At this time the windowed pulse has not yet reached the dielectric material. Figure 4 depicts the electric field at $5.0 \mathrm{~ns}$, where there are both reflection and transmission pulses originating from the initial pulse at $\mathrm{z}=0$. In both cases there is good agreement between the FEM and POD simulations.

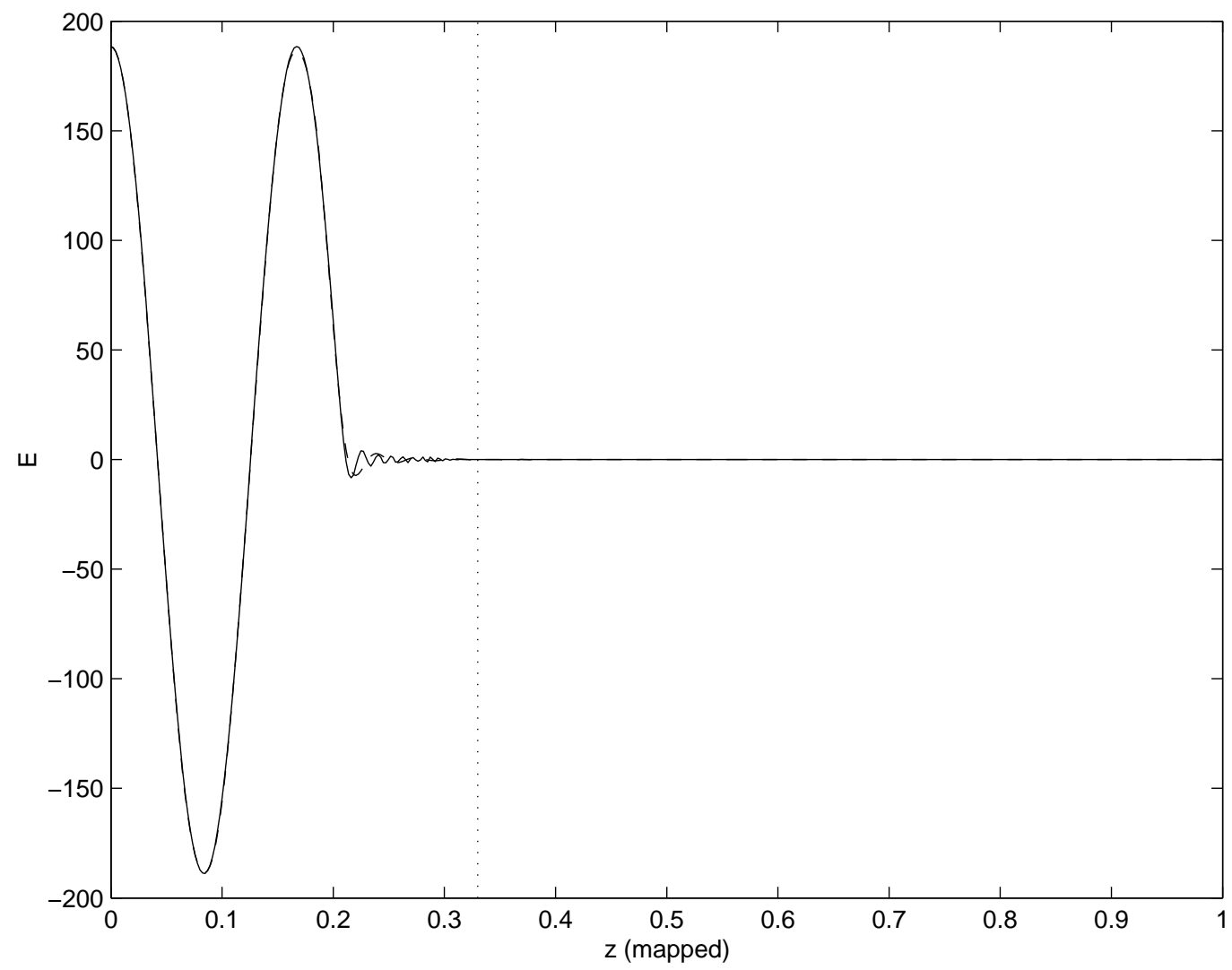

Figure 3: The electric field at $0.7 \mathrm{~ns}$ as a function of $z$ (mapped) for the FEM simulation (solid line) and POD simulation (dash-dot line). The POD reduced order model used 70 modes. 


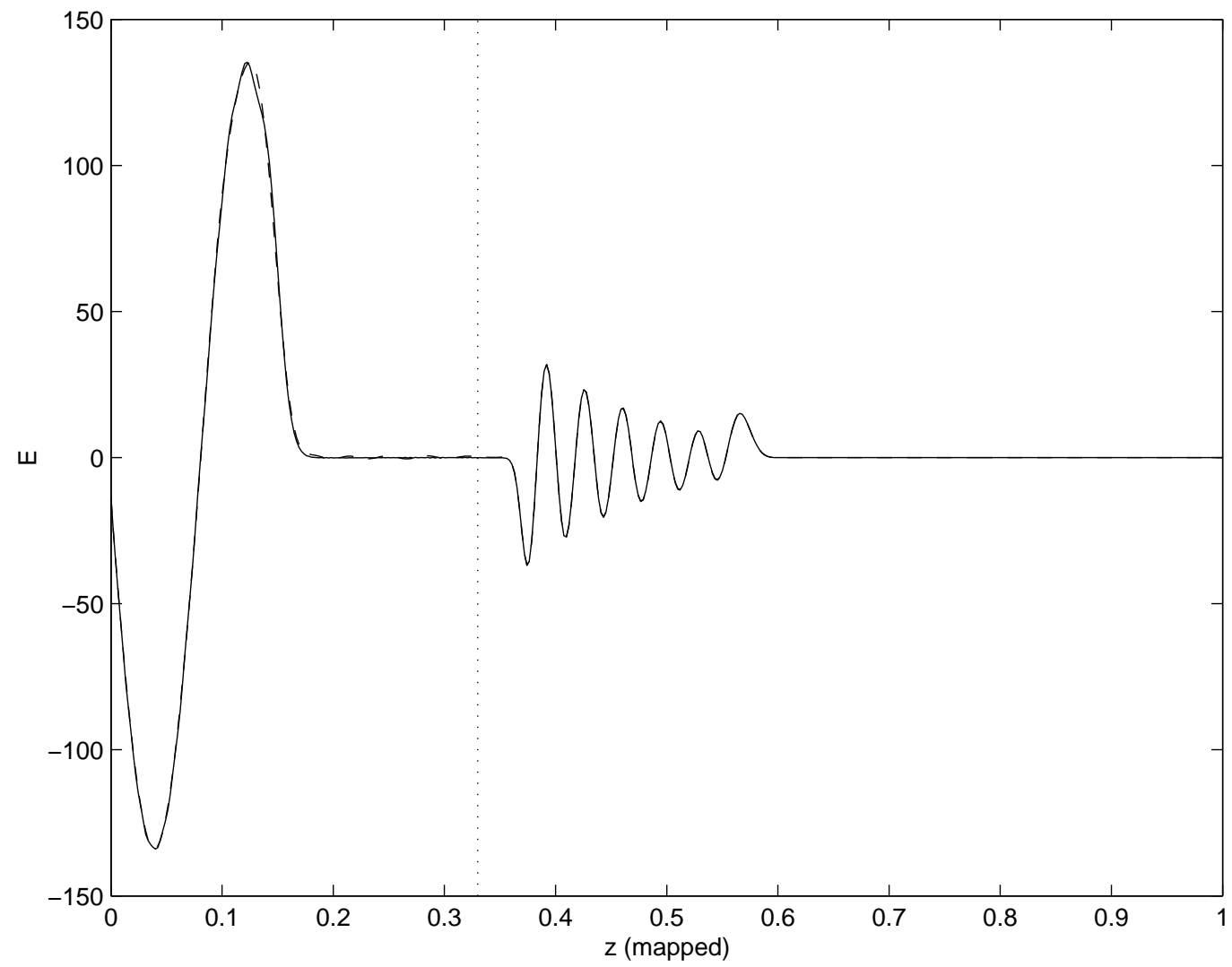

Figure 4: The electric field at $5.0 \mathrm{~ns}$ as a function of $z$ (mapped) for the FEM simulation (solid line) and POD simulation (dash-dot line). The POD reduced order model used 70 modes. 
Figures 5 and 6 show the electric field at 7.0 and 10.0 ns. At these times, only the transmitted portion of the initial pulse remains. The discrepancies between the FEM and POD simulations are more evident due to an increase in relative error because of the very small magnitude of the electric field outside the dielectric material. The Brillouin precursors are evident from the large amplitudes of the forward and trailing peaks in both figures. There is still excellent agreement between the FEM and POD simulations.

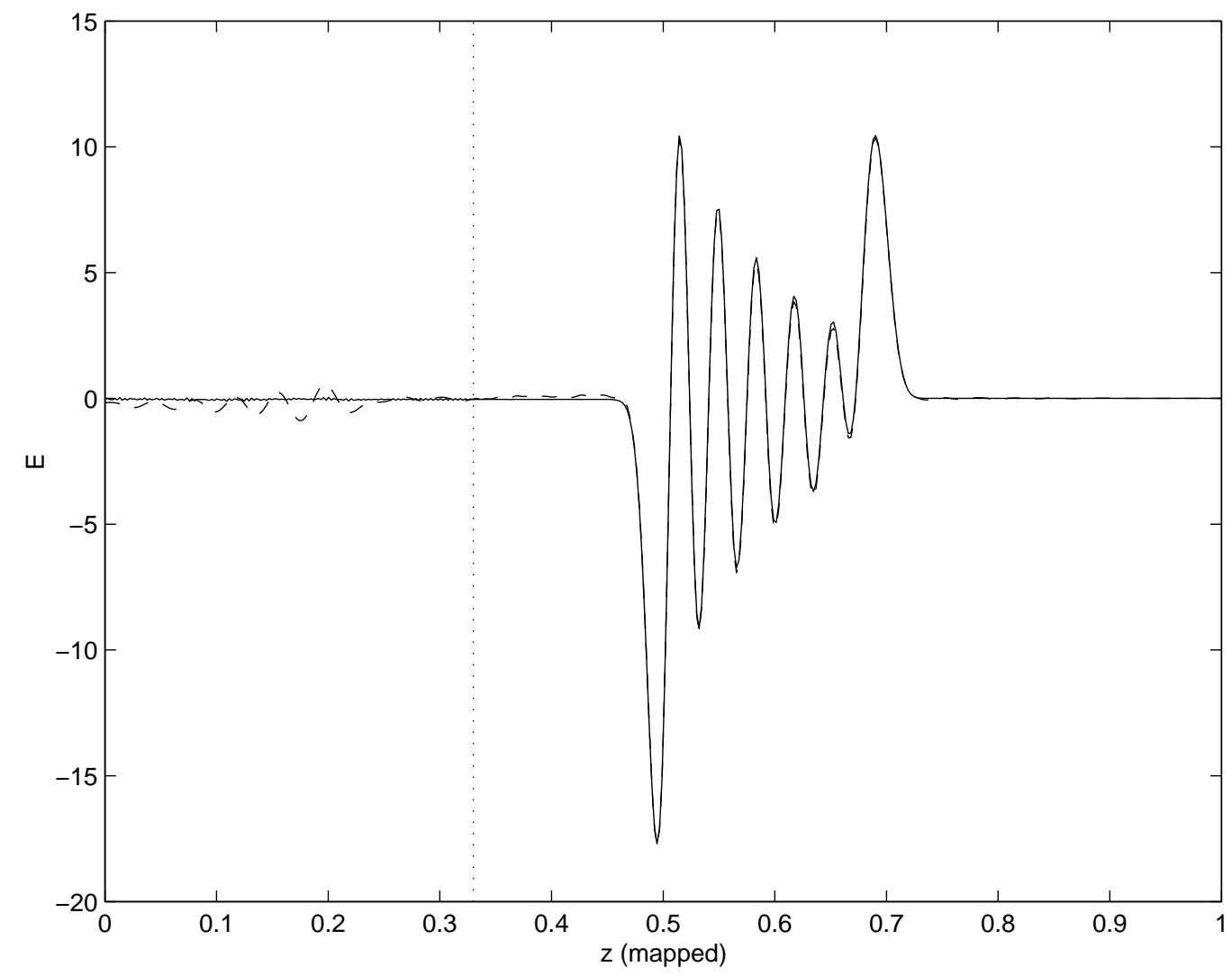

Figure 5: The electric field at $7.0 \mathrm{~ns}$ as a function of $z$ (mapped) for the FEM simulation (solid line) and POD simulation (dash-dot line). The POD reduced order model used 70 modes. 


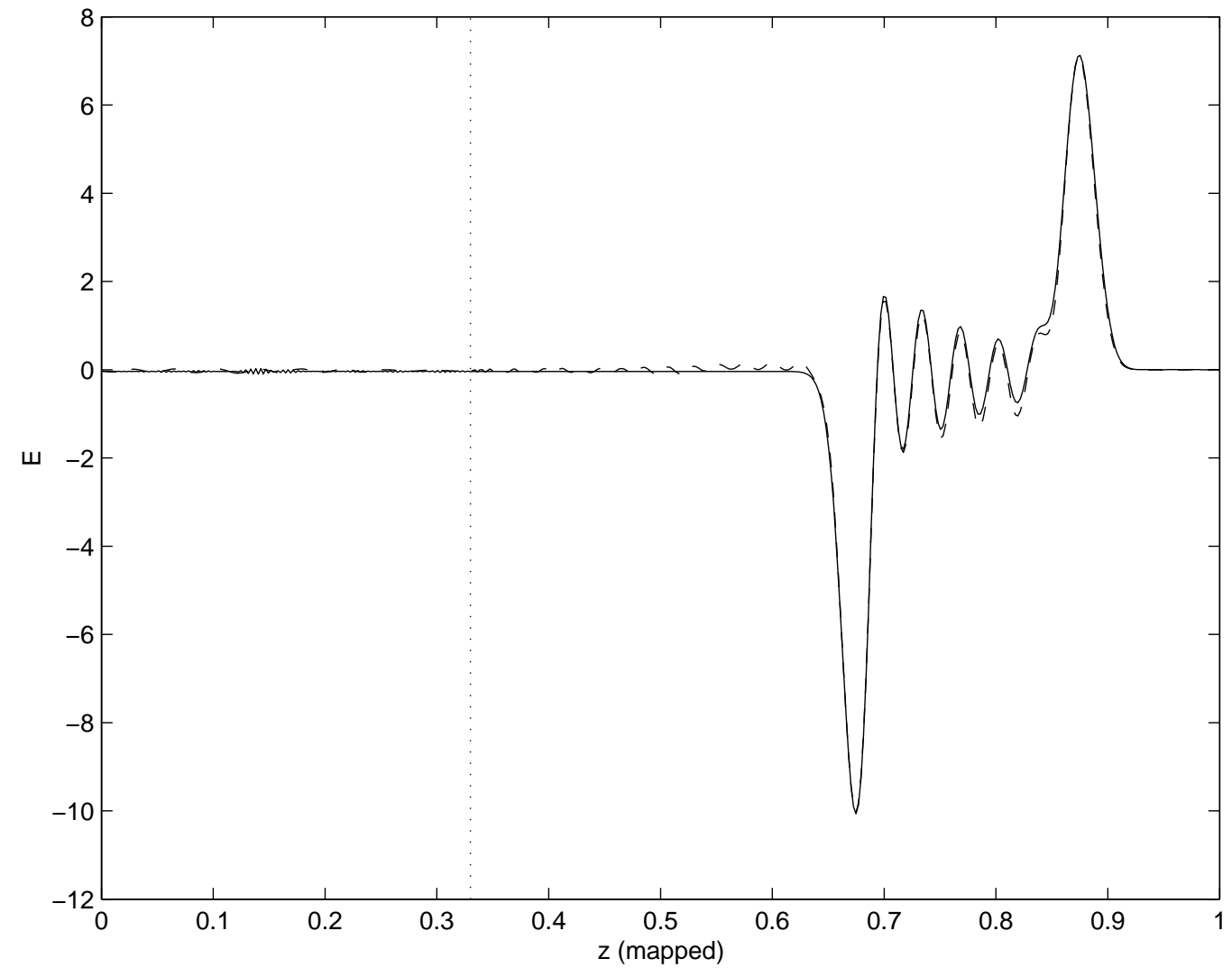

Figure 6: The electric field at $10.0 \mathrm{~ns}$ as a function of $z$ (mapped) for the FEM simulation (solid line) and POD simulation (dash-dot line). The POD reduced order model used 70 modes. 
The solution time with the POD model ( $5 \mathrm{~min}$ ) is significantly less than the solution time required for the 450 node FE model $(272 \mathrm{~min}$ ). Since the efficiency of the modes is relatively independent of the number of nodes used, the time savings of the POD reduced order model over the FE model becomes more significant when more nodes are required.

This point is illustrated in Figure 7, where it can be seen that the FEM solution time is proportional to the number of nodes squared. Also plotted are the equivalent POD solution times, which are calculated in the same manner as above. That is, for each $N$-noded FEM simulation (the parameter values are the same as above, except $t_{\text {final }}=5.0 \mathrm{~ns}$ ) the POD method is applied to snapshots at $0.005 \mathrm{~ns}$ intervals. The simulation time using the POD formulation with $M$ modes (chosen to capture at least $99.999 \%$ of the variability) is the equivalent POD solution time. Figure 7 shows that the equivalent POD solution times are relatively independent of the number of FEM nodes.

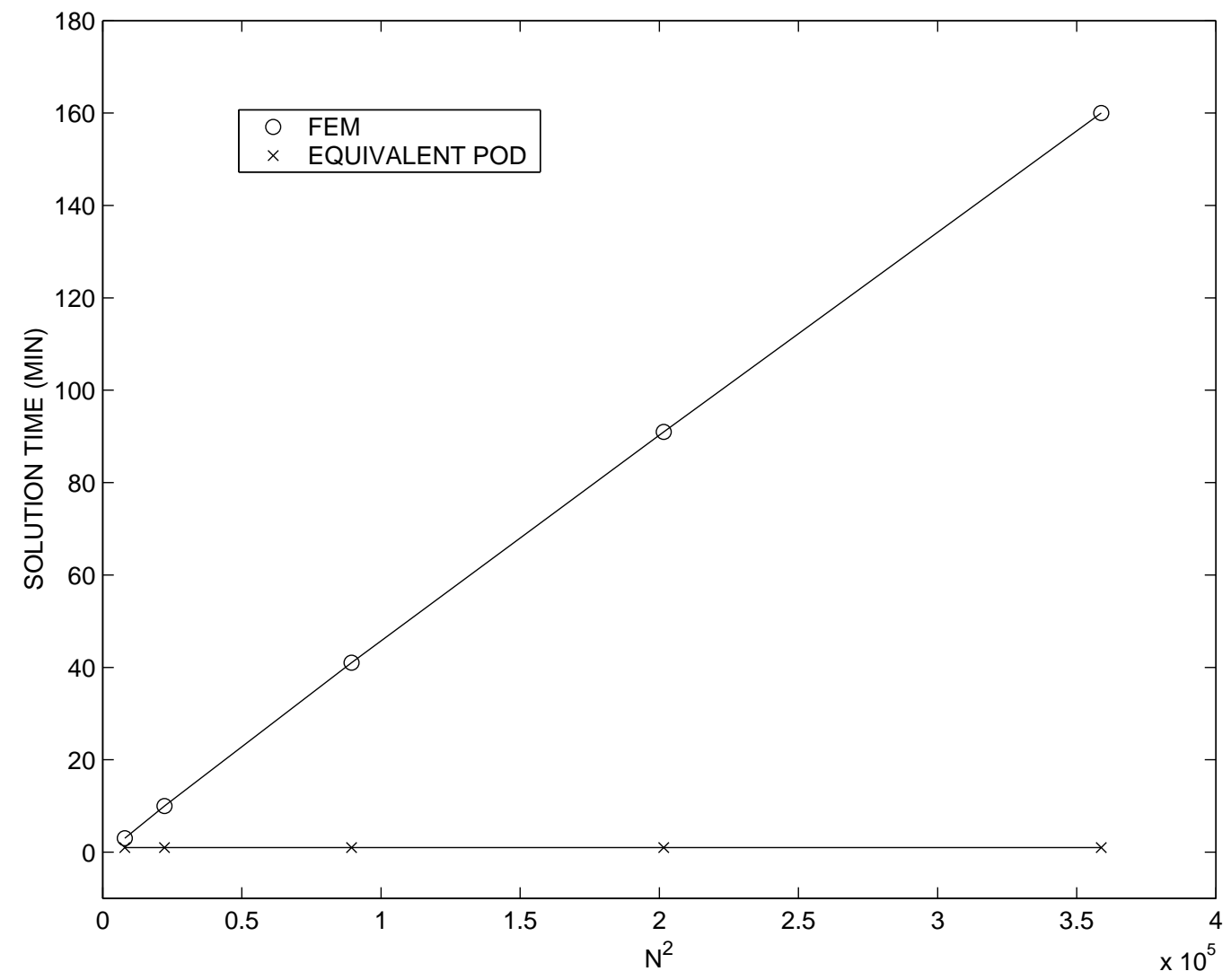

Figure 7: The solution time as a function of the number of nodes squared for the FEM (o) with $t_{\text {final }}=5.0$ ns. Also shown are the equivalent POD solution times (x), where the number of modes captures at least $99.999 \%$ of the data variability. In both cases, data points are connected by straight lines. 
Inverse problem: Example I. We turn to computational findings for a one parameter $\left(\epsilon_{s}\right)$ problem of the interrogation of a dielectric slab with a supraconductive backing by a plane-polarized windowed wave. In the inverse problem we attempt to determine the parameter values $\left(\epsilon_{s}\right)$ of the dielectric material from measurements of the electric field at $z=0$. The dielectric slab dimensions and parameters are the same as above in the forward problem, except for $N=150, L=50$, and $t_{f}=\frac{2}{\phi}=1.11 \times 10^{-9}$.

In this example, we investigate the inverse problem for the single unknown parameter $\epsilon_{s}$ in the range $31 \leq \epsilon_{s} \leq 39$. Three FE simulations $\left(\epsilon_{s}=31,33\right.$, and 39) each with $K=501$ snapshots of the $N-1$ nodal values for the electic and polarization fields are used to obtain the POD basis elements for the minimization problem

$$
X_{6 K}^{N-1}=\left\{X_{2 K}^{N-1}\left(\epsilon_{s}=31\right) X_{2 K}^{N-1}\left(\epsilon_{s}=35\right) X_{2 K}^{N-1}\left(\epsilon_{s}=39\right)\right\} .
$$

The elecric field is measured at $0.01 \mathrm{~ns}$ intervals from $0<t<5 \mathrm{~ns}$. Figure 8 shows the observed values of the electric field at $z=0$ as a function of time.

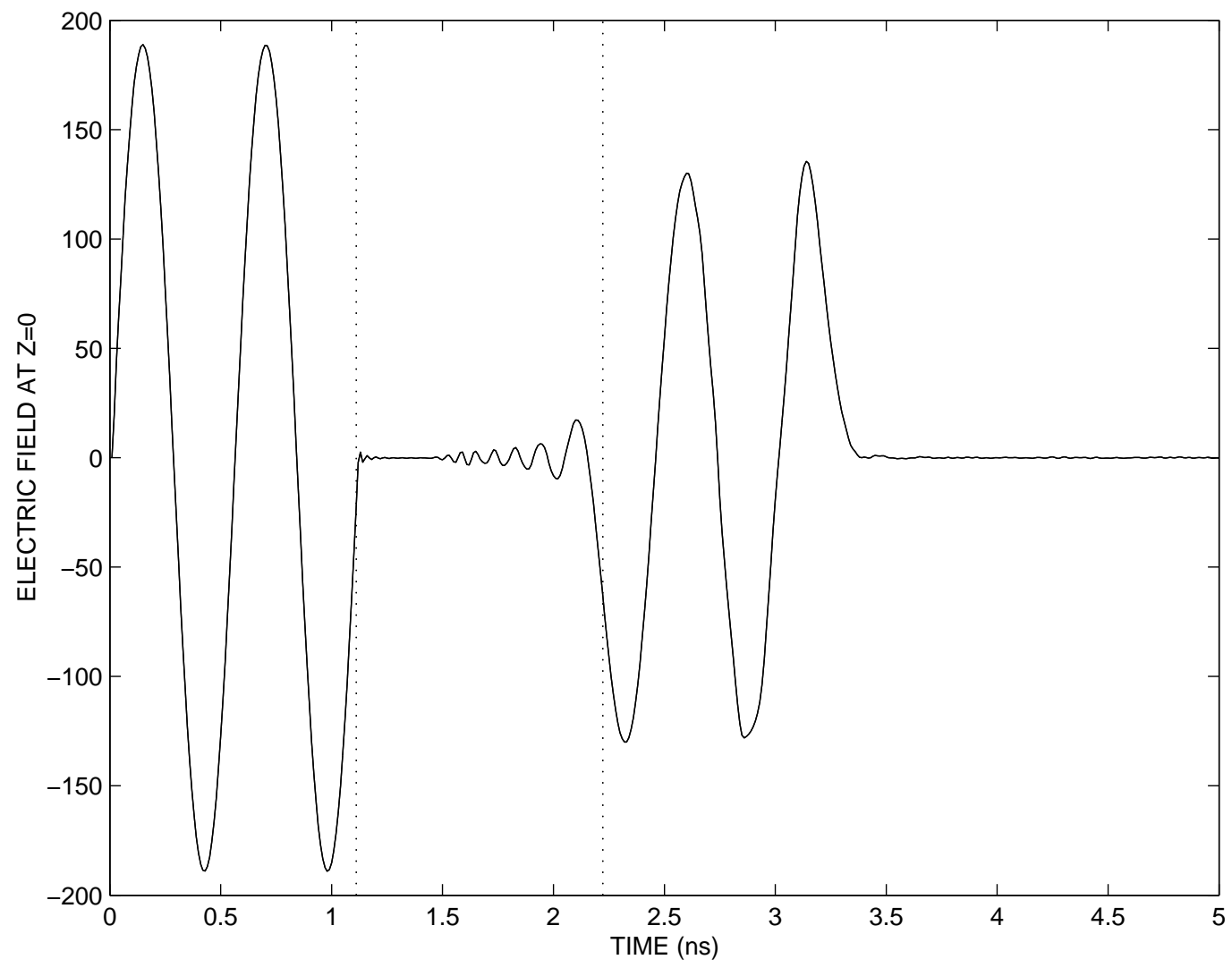

Figure 8: The observed electric field at $\mathrm{z}=0$ as a function of time. Measurements are made at $0.01 \mathrm{~ns}$ intervals from 0 to $5 \mathrm{~ns}$. 
The ability of the POD formulation to recover the true values in the inverse problem is investigated as a function of the number of modes used (Fig. 9). The following conditions were used to obtain these results: $0 \%$ noise, $\epsilon_{s}$ (true $)=33, \epsilon_{s}$ (initial) $=37$, and $J(\vec{q})$ minimized with respect to observation measurements from times $0 \leq t \leq 3.5 \mathrm{~ns}$. As expected, the relative error generally decreases as the number of modes increases. Oscillations may be due to the influence of the addition of particular modes with respect to the true parameter value. As Fig. 9 indicates, the relative error decreases slowly after approximately 50 modes.

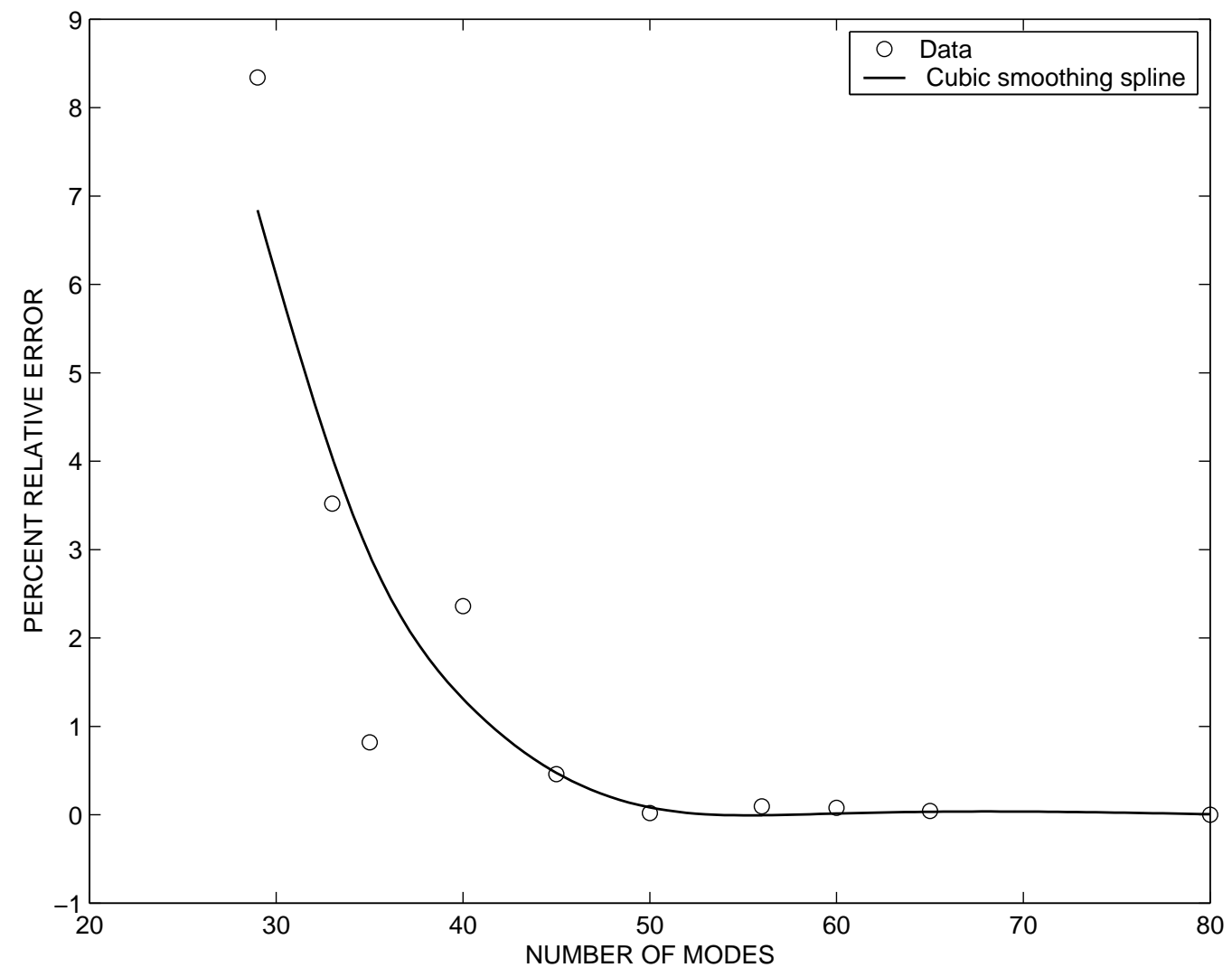

Figure 9: The percent relative recovered parameter error $\left(100 \mid \epsilon_{s}(\right.$ true $)-\epsilon_{s}($ recovered $\left.)\right) / \epsilon_{s}($ true $\left.) \mid\right)$ as a function of the number of modes (circles). The curve drawn through the data is a cubic smoothing spline (csaps in Matlab) with smoothing parameter equal to 0.1 
We next investigated the ability of the POD formulation to recover $\epsilon_{s}$ with a noisy signal and a fixed number of modes (60). Figure 10 gives an example of the observed electric field without noise and with $5 \%$ noise added. In this case $\epsilon_{s}=33$.

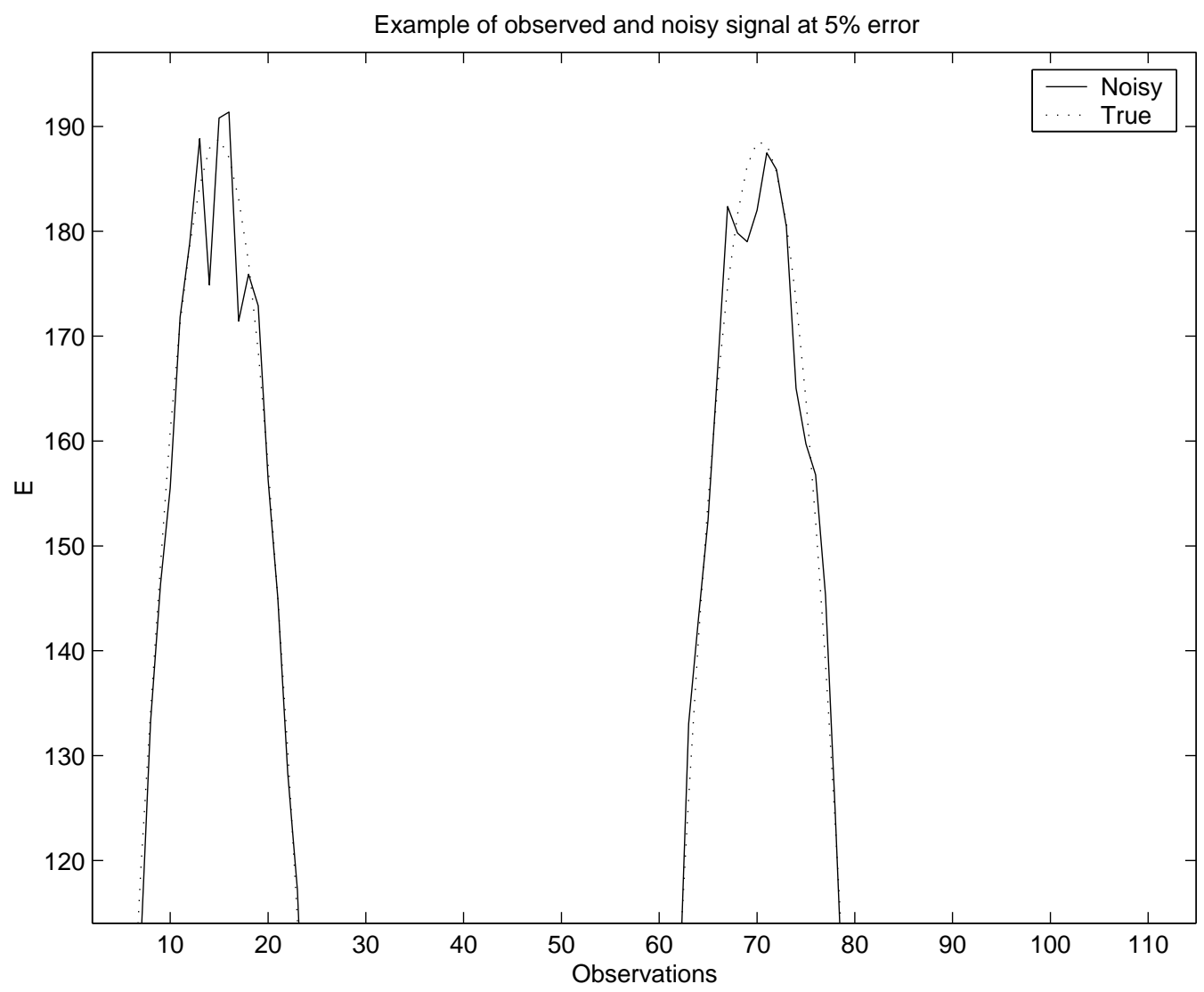

Figure 10: A portion of the observed electric field at $\mathrm{z}=0$ with $5 \%$ noise (solid line) and without added noise (dashed line), for $\epsilon_{s}$ (true) $=33$. 
The accuracy of the POD formulation in the above inverse problem with one unknown parameter $\left(\epsilon_{s}\right)$ is compared to the FEM formulation in Fig. 11, where the relative error is plotted as a function of the percent noise in the signal. For basis of comparison, the same seed (-123456) for the random number generator is used for all cases with non-zero noise. In general the relative error increases linearly with respect to the percent noise for both the POD and FEM formulations. Except at the zero percent noise level, the POD method is slightly more accurate than the FEM formulations. We attribute this to serendipity rather than any remarkable methodological principle.

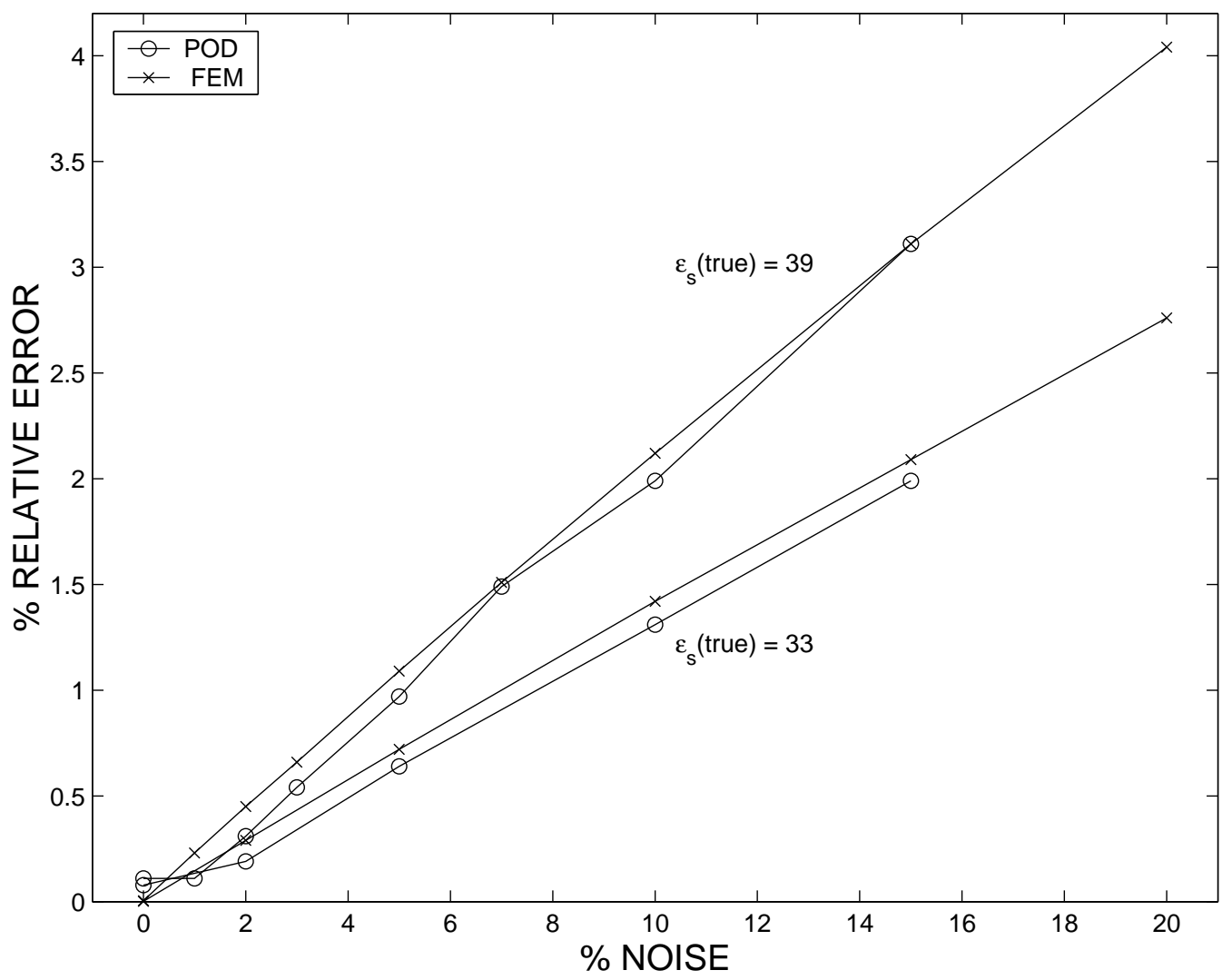

Figure 11: The percent relative recovered parameter error $\left(100 \mid \epsilon_{s}(\right.$ true $)-\epsilon_{s}($ recovered $\left.)\right) / \epsilon_{s}($ true $\left.) \mid\right)$ as a function of the percent noise for the POD (o, 60 modes) and FEM (x) formulations. In both case data points are connected by straight lines.

A plot of the ratio of FEM solution times to POD solution times illustrates the time savings offered by the POD method. As Fig. 12 indicates, the POD method is most efficient at the $0 \%$ noise level where it is nine times faster than the FEM formulation. As the noise level increases the POD efficiency decreases, eventually reaching a 5.4-fold time saving at the $15 \%$ noise level. The POD times are not changing much as the noise level changes, but the FEM solution times decrease somewhat. 


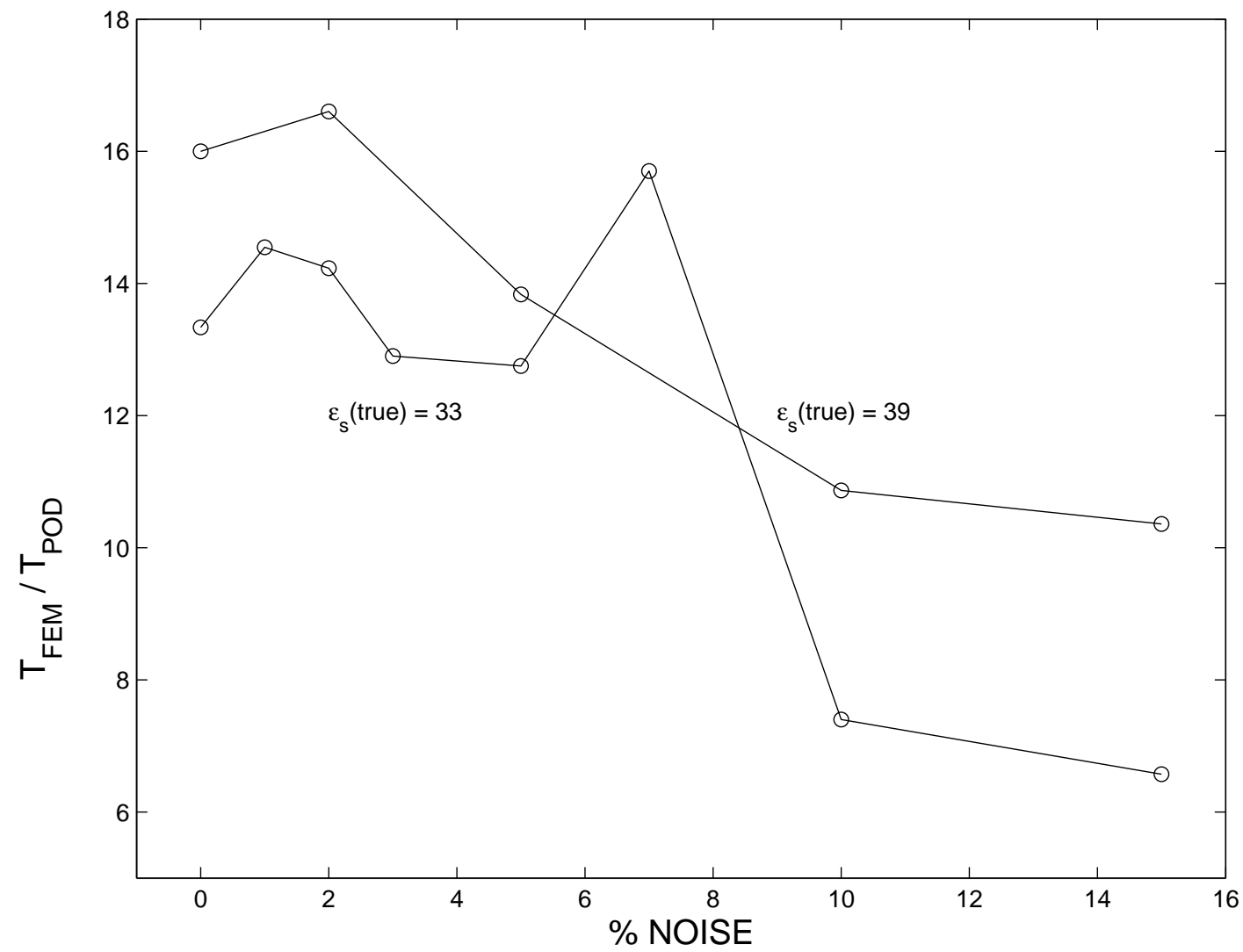

Figure 12: The ratio of solution times $\left(\mathrm{T}_{F E M} / \mathrm{T}_{P O D}\right)$ as a function of the percent noise. The data points are connected by straight lines.

Inverse problem: Example II. In this example, we investigate the inverse problem with $\tau$ as the single unknown parameter. The problem conditions are the same as in the previous example, except that now we employ fixed $\epsilon_{s}=35$ and $\tau$ is varied. Three FE simulations $\left(\tau=1 \times 10^{-12}, 1 \times 10^{-11}\right.$ and $\left.1 \times 10^{-10}\right)$ each with $K=501$ snapshots of the $N-1$ nodal values for the electic and polarization fields are used to obtain the POD basis elements for the minimization problem

$$
X_{6 K}^{N-1}=\left\{X_{2 K}^{N-1}\left(\tau=1 \times 10^{-12}\right) X_{2 K}^{N-1}\left(\tau=1 \times 10^{-11}\right) X_{2 K}^{N-1}\left(\tau=1 \times 10^{-10}\right)\right\} .
$$

The ability of the POD formulation to recover the true values in the inverse problem is plotted as a function of the number of modes used in Fig. 13. The following conditions were used in these results: $0 \%$ noise, $\tau($ true $)=5 \times 10^{-11}, \tau($ initial $)=3.5 \times 10^{-11}$, and $J(\vec{q})$ minimized with respect to observation measurements from times $0 \leq t \leq 3.5 \mathrm{~ns}$. The relative error generally decreases as the number of modes increases. As in the previous example, oscillations may be due to the influence of the addition of particular modes with respect to the true parameter value. The relative error decreases slowly after approximately 40 modes, where it is already under $1 \%$. 


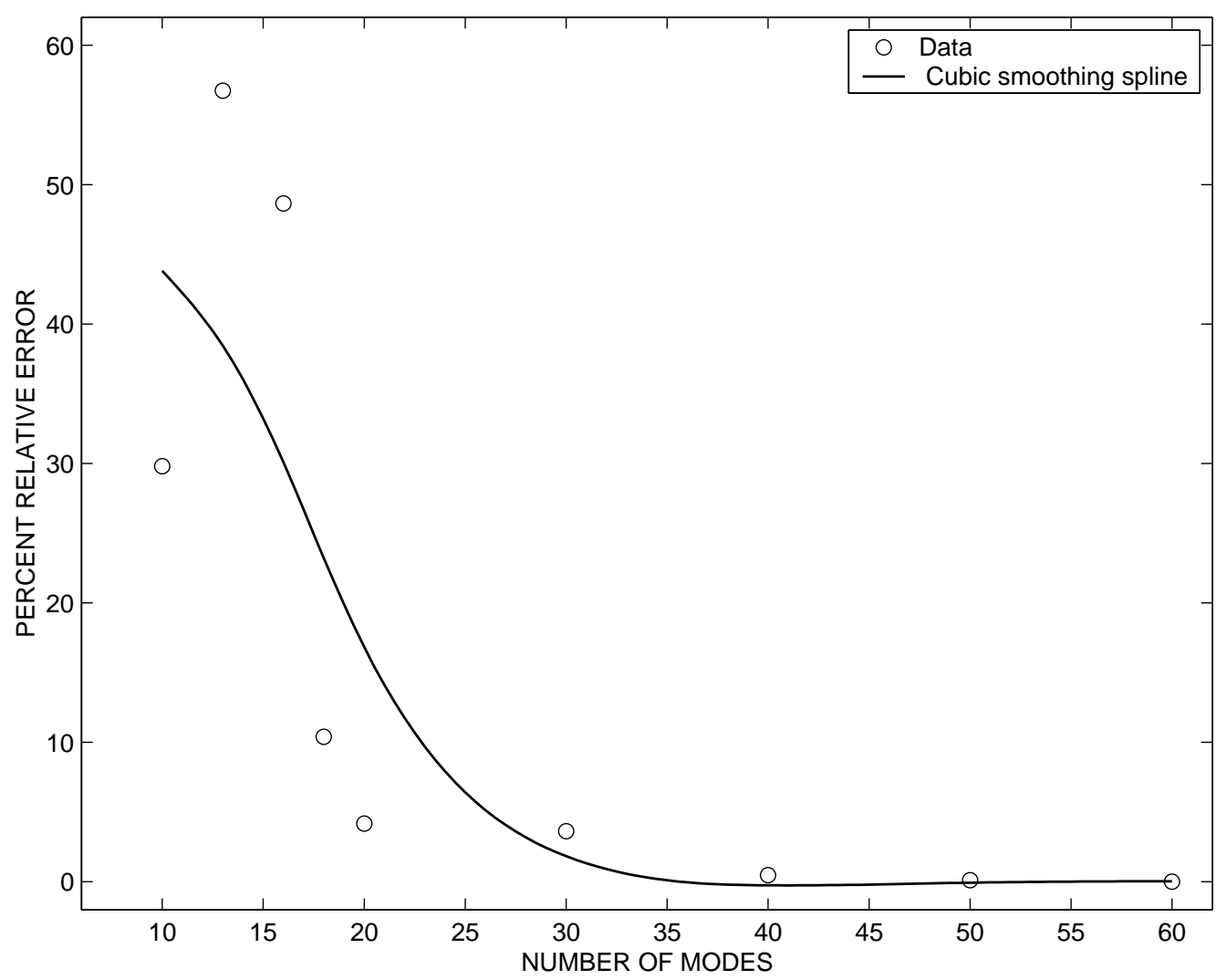

Figure 13: The percent relative recovered parameter error $(100 \mid \tau($ true $)-\tau($ recovered $)) / \tau($ true $) \mid)$ as a function of the number of modes (circles). The curve drawn through the data is a cubic smoothing spline (csaps in Matlab) with smoothing parameter equal to 0.1

The accuracy of the POD formulation with $\tau$ unknown is compared to the FEM formulation in Fig. 14, where the relative error is plotted as a function of the percent noise in the signal. For basis of comparison, the same seed (-123456) for the random number generator is used for all cases with non-zero noise. As expected, the relative error increases with respect to the percent noise for both the POD and FEM formulations. The FEM method is more accurate than the POD at low noise levels. However, above the $5 \%$ noise level the POD method is increasingly slightly more accurate than the FE method. 


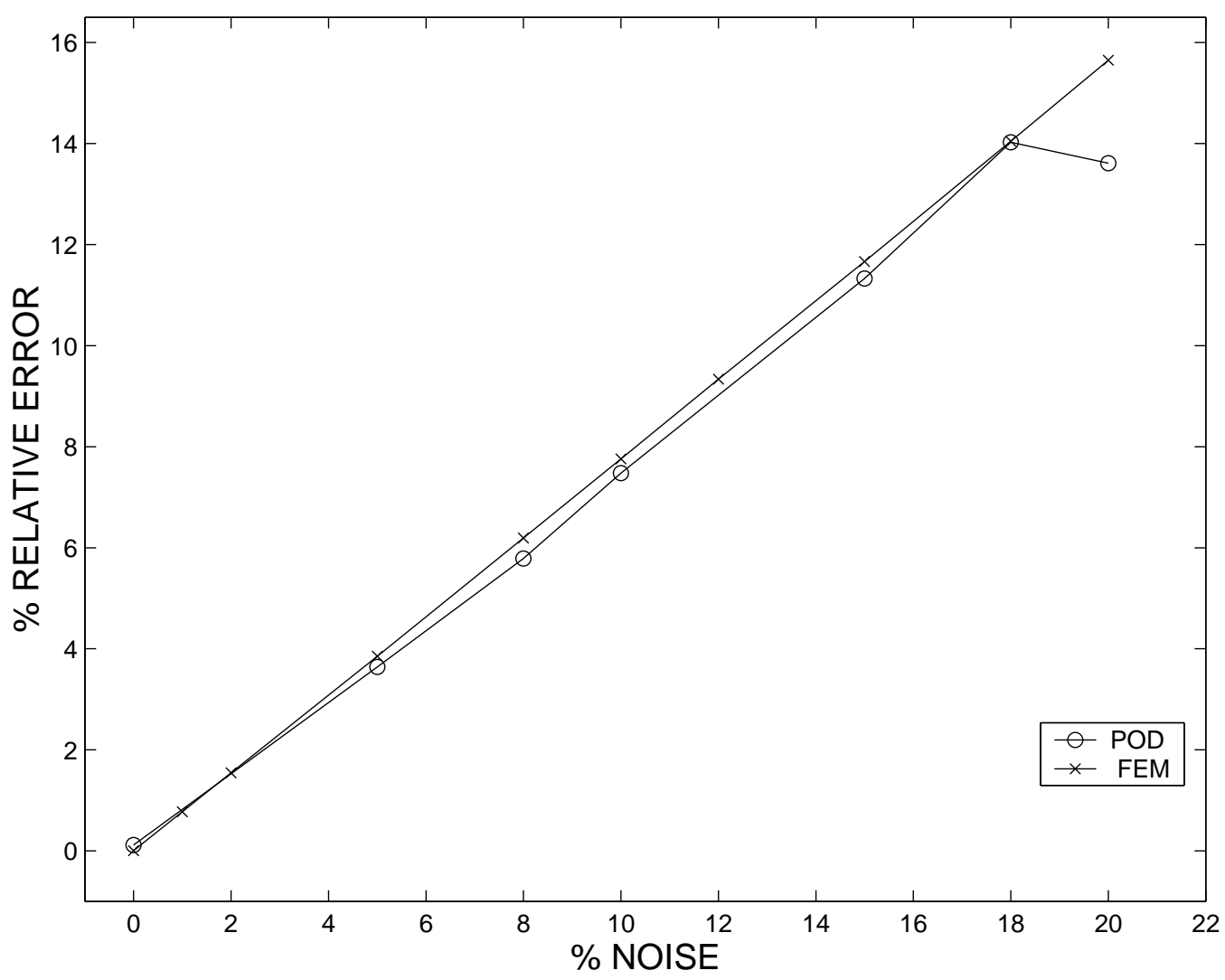

Figure 14: The percent relative recovered parameter error $(100 \mid \tau($ true $)-\tau($ recovered $)) / \tau($ true $) \mid)$ as a function of the percent noise for the POD (o, 60 modes) and FEM (x) formulations. In both case data points are connected by straight lines.

A plot of the ratio of FEM solution times to POD solution times illustrates the time savings offered by the POD method. As Fig. 15 indicates, the POD method is 4.5 to 8 times more efficient than the FEM formulation. Variations in the efficiency are largely due to variations in the solution times for the FEM. 


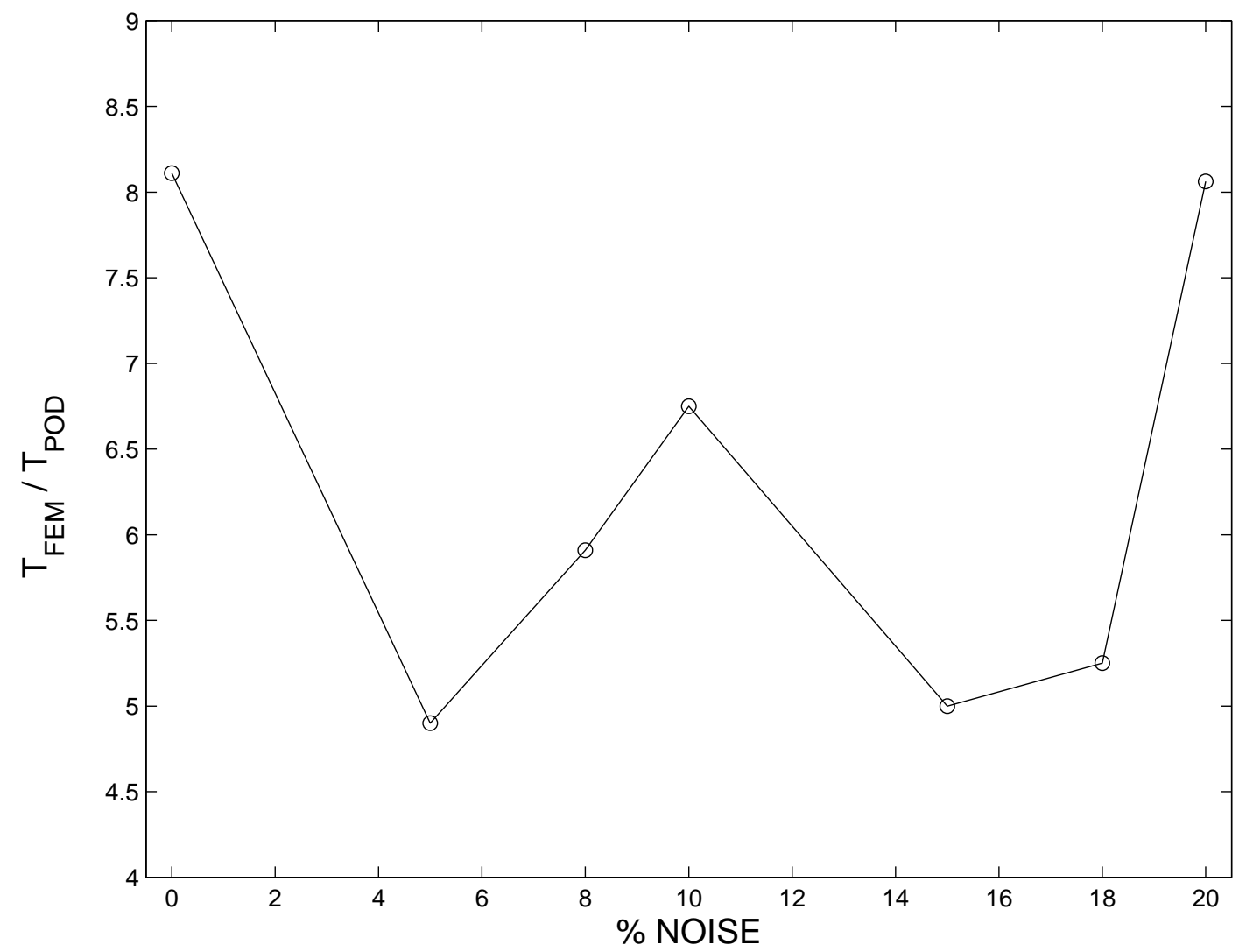

Figure 15: The ratio of solution times $\left(\mathrm{T}_{F E M} / \mathrm{T}_{P O D}\right)$ as a function of the percent noise. The data points are connected by straight lines.

Inverse problem: Example III. In this example both parameters $\epsilon_{s}$ and $\tau$ are simultaneously sought in a two parameter inverse problem. Except for $\epsilon_{s}$ and $\tau$, simulation conditions were the same as in in the one parameter inverse problems above. The elecric field is measured at $0.01 \mathrm{~ns}$ intervals from $0<t<5$ ns. Nine FE simulations (combinations of $\epsilon_{s}=31,33,39$ and $\tau=1 \times 10^{-12}, 1 \times 10^{-11}, 1 \times 10^{-10}$ ) each with $K=501$ snapshots of the $N=149$ nodal values for the electic and polarization fields are used to obtain the POD basis elements for the minimization problem.

Figures 16 and 17 summarize the two parameter inverse results under the following conditions $\epsilon_{s}($ true $)=33$, $\epsilon_{s}($ initial $)=37, \tau($ true $)=5 \times 10^{-11}, \tau($ initial $)=3.5 \times 10^{-11}$, and $J(\vec{q})$ minimized with respect to observation measurements from times $0 \leq t \leq 3.5$ ns.

Figure 16 plots the relative error for the recovered $\epsilon_{s}$ values as a function of the noise level (0-20\%) for the POD and FEM methods. From Fig. 16 it can be seen that the FEM results are generally more accurate than the POD results, but that the relative error for both is under $0.2 \%$. The relationship between the relative error and the noise level appears to be nonlinear, unlike the one parameter recovery of $\epsilon_{s}$. 


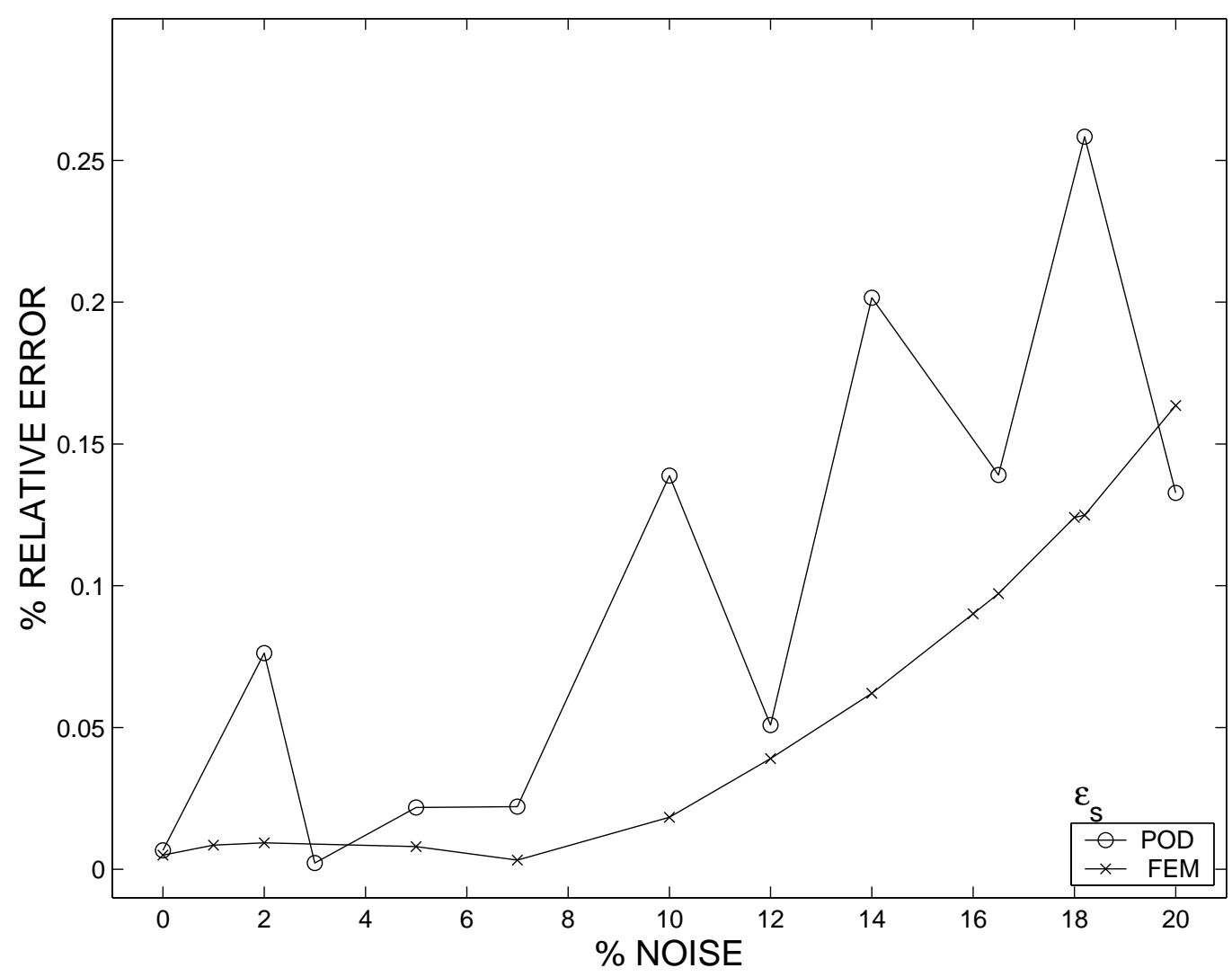

Figure 16: The percent relative recovered parameter error $\left(100 \mid \epsilon_{s}(\right.$ true $)-\epsilon_{s}$ (recovered $\left.)\right) / \epsilon_{s}($ true $\left.) \mid\right)$ as a function of the percent noise for the POD (o, 50 modes) and FEM (x) formulations. Data points are connected by straight lines.

Figure 17 plots the relative error for the recovered $\tau$ values as a function of the noise level (0-20\%) for the POD and FEM methods. The relative error appears to be roughly linear with respect to the noise level for both POD and FEM formulations. The relative error for both formulations do not differ greatly except at the $20 \%$ noise level. The relative error for recovery of $\tau(0-16 \%)$ is about ten times greater than the relative error associated with the recovery of $\epsilon_{s}(0-0.16 \%)$. (This agrees with relative sensitivity of the model to the parameters seen in [10].) 


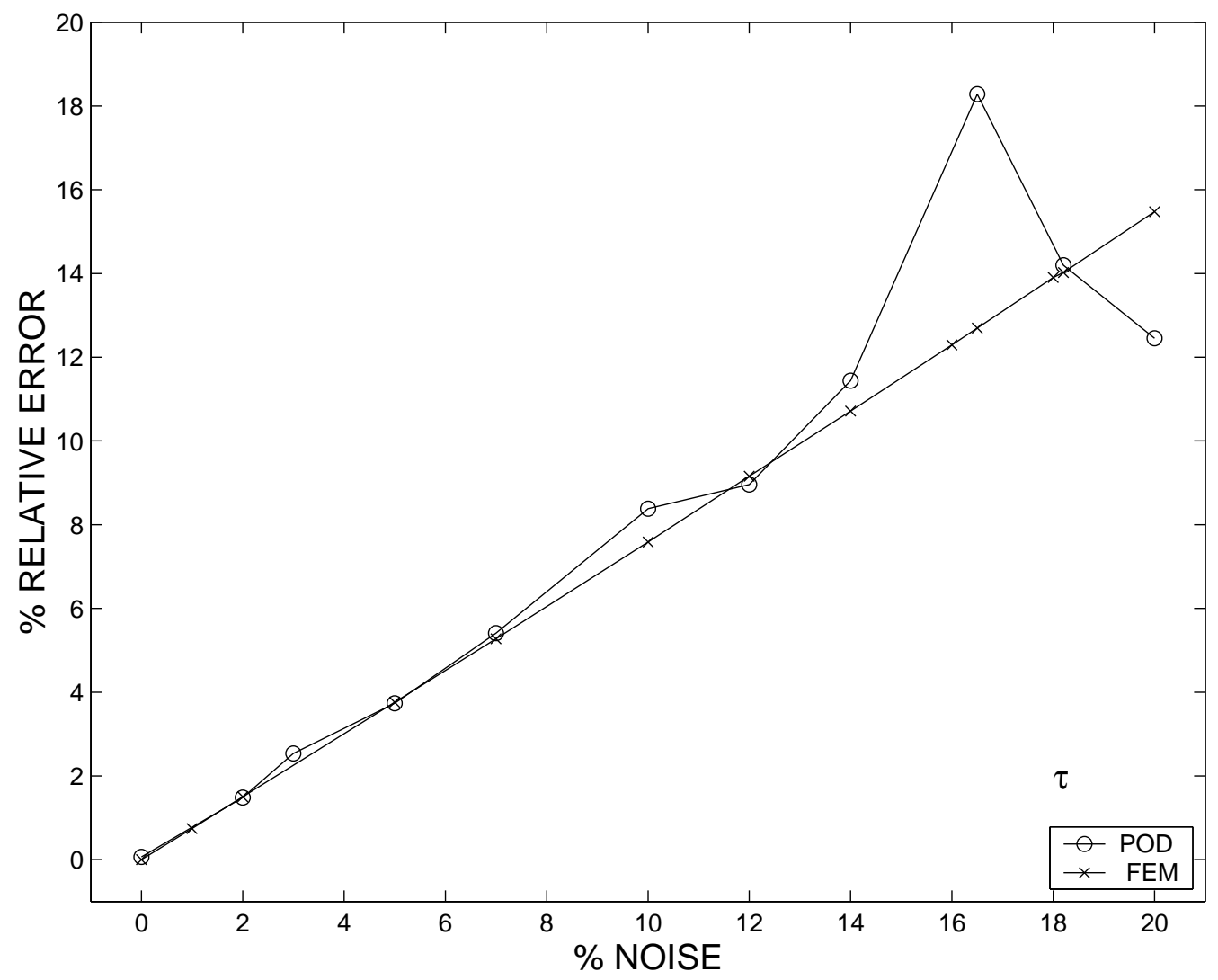

Figure 17: The percent relative recovered parameter error $(100 \mid \tau($ true $)-\tau$ (recovered $)) / \tau($ true $) \mid)$ as a function of the percent noise for the POD (o, 50 modes) and FEM (x) formulations. Data points are connected by straight lines.

Figure 18 plots the ratio of the FEM simulation times and the POD simulation times as a function of noise (0-20\%). The POD formulation is 4-10 times faster than the FE method. The time savings generally decrease as the noise level rises. 


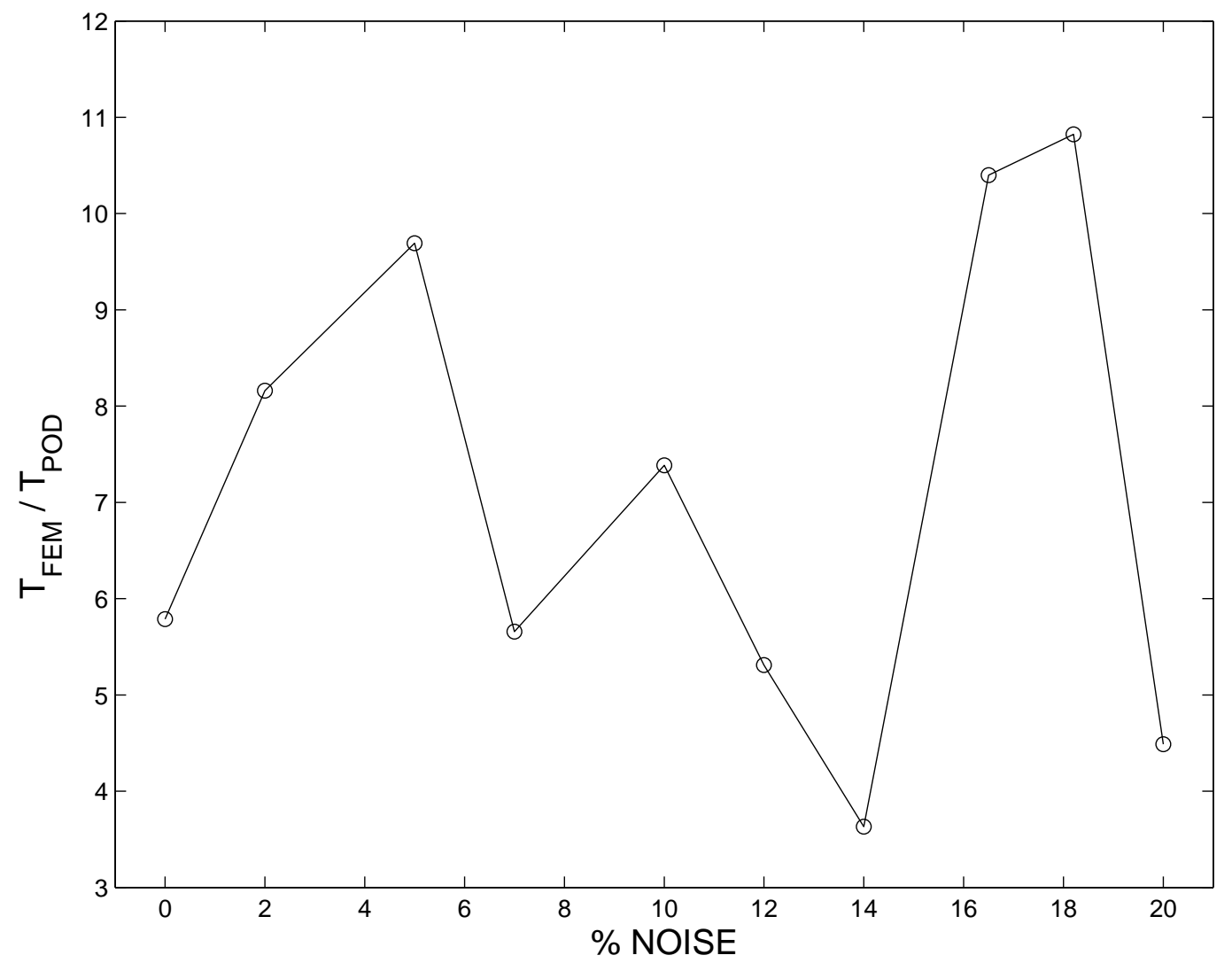

Figure 18: The ratio of solution times $\left(\mathrm{T}_{F E M} / \mathrm{T}_{P O D}\right)$ as a function of the percent noise for the two parameter inverse problem. The data points are connected by straight lines.

\section{REMARKS AND CONCLUSIONS}

The saving in solution time using the reduced order POD formulation instead of the FEM formulation is most evident as the number of nodes increases. The solution times scales as $N^{2}$, where $N$ is the number of FEM nodes or POD modes. However, the number of modes necessary to capture the physical behavior is relatively independent of the number of nodes used to generate the data.

The POD method is significantly faster than the FEM method for inverse problems. The POD method (50 modes) is 4-10 times faster than the FEM (149 nodes) in the two parameter $\left(\epsilon_{s}\right.$ and $\tau$ ) inverse problem and in the single parameter $\left(\epsilon_{s}\right.$ or $\left.\tau\right)$ inverse problem. Based on our results from the forward simulation problems (where savings using POD over FEM dramatically increased as $\mathrm{N}$ increased, e.g., 50 fold speed up for $N=450$ ), we expect the speed up in inverse problems to dramatically increase in more complex problems (e.g., 2-D and 3-D problems) where an increasing number of basis elements are required.

One of the difficulties with using the POD method for inverse problems is in the construction of the POD modes. Memory and time limitations become significant as the number of parameters is increased in a time-dependent problem such as reported here. If, in a $N$-noded simulation, a time series of $(M)$ 
snapshots is generated at three representative values spanning the desired range of each parameter, there are a total of $3^{p} M N$ snapshots. On the PC used for these calculations there is a memory limitation of approximately 6000 snapshots $(N=150)$ in the algorithm used to construct the POD modes. As an example of the time-limitiation, construction of POD modes for the case of $N=150$ and 5010 snapshots required 18 hours and 20 minutes to complete. Current efforts and ideas to alleviate these aspects of difficulties are being pursued by others as well as our group at NCSU.

The findings in this paper offer great potential and encouragement for our ongoing efforts in higher dimensional problems where a number of significant computational difficulties might be alleviated by use of a reduced order methodology for transient electromagnetic inverse problems.

\section{$5 \quad$ ACKNOWLEDGMENTS}

This research was supported in part by the U.S. Air Force Offices of Scientific Research under grant AFOSR F49620-01-1-0026. Part of the research was facilitated by efforts while the authors were in residence at the Statistical and Applied Mathematical Sciences Institute (SAMSI) in RTP which is supported by the NSF and a consortium of Duke, NCSU, UNC-CH, and NISS. 


\section{References}

[1] R.A. Albanese, R.L. Meina, and J.W. Penn, "Mathematics, medicine and microwaves", Inverse Problems 10, 995-1007, 1994.

[2] J.A. Atwell and B. King, "Proper orthogonal decomposition for reduced basis feedback controllers for parabolic equations", VPISU, Blacksburg VA, ICAM Rep. 99-01-01, Jan. 1999; Math. and Comput. Modeling, to appear.

[3] J.A. Atwell, and B. King, "Reduced order controllers for spatially distributed systems via proper orthogonal decomposition", VPISU, Blacksburg VA, ICAM Rep. 99-07-01, July 1999.

[4] N. Aubry, P. Holmes, J.L. Lumley, and E. Stone, "The dynamics of coherent structures in the wall region of a turbulent boundary layer", Journal of Fluid Mechanics 192, 115-173, 1988.

[5] H.T. Banks and F. Kojima, "Boundary shape identification problems in two dimensional domains related to thermal testing of materials", Quart. Appl. Math 47, 273-293, 1989.

[6] H.T. Banks, F. Kojima, and W.P. Winfree, "Boundary estimation problems arising in thermal tomography", Inverse Problems 6, 897-921, 1990.

[7] H.T. Banks, R.C.H. del Rosario, and R.C. Smith, "Reduced order model feedback control design: Numerical implementation in a thin shell model", N.C. State University, Raleigh, NC, CRSC Tech Rep, 98-27, July 1998; IEEE Trans. Auto. Control, 45, 1312-1324, 2000.

[8] H.T. Banks, R.C.H. del Rosario, and R.C. Smith, "Reduced order model feedback control design: Computational studies for thin cylindrical shells", N.C. State University, Raleigh, NC, CRSC Tech Rep, 98-25, June 1998.

[9] H.T. Banks, M.L. Joyner, B. Wincheski, and W.P. Winfree, "Nondestructive evaluation using a reduced-order computational methodology", Inverse Problems 16, 929-945, 2000

[10] H.T. Banks, M.W. Buksas, and T. Lin, Electomagnetic Material Interrogation Using Conductive Interfaces and Acoustic Wavefronts, SIAM, Philadelphia, 2000.

[11] H.T. Banks, M.L. Joyner, B. Wincheski, and W.P. Winfree, "Real time computational algorithms for eddy-current-based damage detection", Inverse Problems 18, 795-823, 2002

[12] H.T. Banks, S.C. Beeler, G.M. Kepler, and H.T. Tran, "Reduced order modeling and control of thin film growth in a HPCVD reactor", SIAM Journal of Applied Mathematics 62, 1251-1280, 2002.

[13] G. Berkooz, "Observations on the proper orthogonal decomposition", in Studies in Turbulence, T.B. Gatski, S. Sarkar, and C.G. Speziale, Eds. New York: Springer-Verlag, 1992, 229-247.

[14] G. Berkooz, P. Holmes, and J.L. Lumley, "The proper orthogonal decomposition in the analysis of turbulent flows", Annual Review of Fluids Mechanics 25, N5:539-575, 1993.

[15] G. Berkooz, P. Holmes J.L. Lumley, and J.C. Mattingly, "Low-dimensional models of coherent structures in turbulence", Physics Reports-Review Section of Physics Letters 287, N4:338-384, 1997.

[16] D.H. Chambers, R.J. Adrian, P. Moin, D.S. Stewart, and H.J. Sung, "Karhunen-Loève expansion of Burgers' model of turbulence", Phys. Fluids 31, 2573-2582, 1988.

[17] P. Debye, Polar Molecules, Chemical Catalog Co., New York, 1929.

[18] K. Fukunaga, Introduction to Statistical Pattern Recognition, Academic Press, New York, 1972. 
[19] A. Iollo, S. Lanteri, and J.A. Désidéri, "Stability properties of POD-Galerkin approximations for the compressible Navier-Stokes equations", Sophia Antipolis, INRIA Rep. de Rech., no. 3589, December, 1998.

[20] J.D. Jackson, Classical Electrodynamics, 2nd edition, John Wiley, New York, 1975.

[21] J.E. Jackson, A User's Guide to Principal Components, Wiley, New York, 1991.

[22] K. Karhunen, "Zur spektral theorie stochasticher prozesse", Ann. Acad. Sci. Fennicae Ser. A1 Math Phys. 37, 1-79, 1946.

[23] G.M. Kepler, H.T. Tran, and H.T. Banks, "Compensator control for chemical vapor deposition film growth using reduced order design models", IEEE Transaction on Semiconductor Manufacturing 14, 231-241, 2001.

[24] G.M. Kepler, H.T. Tran, and H.T. Banks, "Reduced order model compensator control of species transport in a CVD reactor", Optimal Control Applications and Methods 21, 143-160, 2000.

[25] K. Kunisch and S. Volkwein, "Control of Burgers' equation by a reduced order approach using proper orthogonal decomposition", J. Opt. Theory Applic. 102, 345-371, 1999.

[26] M. Loève, "Quelques proprietes de fonctions de second ordre", Compte Rend. Acad. Sci. (Paris) 220, 469-470, 1945.

[27] J.L. Lumley, "The structure of inhomogeneous turbulent flows", in Atmospheric Turbulence and Radio Wave Propagation, A.M. Yaglom and V.I. Tatarski, Eds. Moscow: Nauka, 1967, pp. 166-178.

[28] J.L. Lumley, Stochastic Tools in Turbulence, Academic Press, New York, 1970.

[29] H.V. Ly and H.T. Tran, "Proper orthogonal decomposition for flow calculations and optimal control in a horizontal CVD reactor", N.C. State University, Raleigh, NC, CRSC Tech Rep 98-13, March 1998; Quarterly of Applied Mathematics, to be published.

[30] H.V. Ly and H.T. Tran, "Modeling and control of physical processes using proper orthogonal decomposition", Math Comp Model 33, 223-236, 2001.

[31] O. Pironneau, Optimal Shape Design for Elliptic Systems, Springer-Verlag, New York, 1983.

[32] W.H. Press, S.A. Teukolsky, W.T. Vetterling, and B.P. Flannery, Numerical Recipes in C. 2nd edition. Cambridge University Press, New York, 1994.

[33] L. Sirovich and M. Kirby, "Low-dimensional procedure for the characterization of human faces", $J$. Opt. Soc. Am. 4, 519-524, 1987.

[34] L. Sirovich, "Chaotic dynamics of coherent structures", Physica D 37, 126-145, 1989.

[35] A. Theodoropolou, R.A. Adomaitis, and E. Zafiriou, "Model reduction for optimization of rapid thermal chemical vapor deposition systems", IEEE Trans. Semiconductor Manuf. 11, 85-98, 1998. 